Kinetic study of electrostatic twisted waves instability in nonthermal dusty plasmas

Kashif Arshad, M. Lazar, Shahzad Mahmood, Aman-ur-Rehman, and S. Poedts

Citation: Physics of Plasmas 24, 033701 (2017); doi: 10.1063/1.4977446

View online: http://dx.doi.org/10.1063/1.4977446

View Table of Contents: http://aip.scitation.org/toc/php/24/3

Published by the American Institute of Physics 


\title{
Kinetic study of electrostatic twisted waves instability in nonthermal dusty plasmas
}

\author{
Kashif Arshad, ${ }^{1,2, a)}$ M. Lazar, ${ }^{1,3, b)}$ Shahzad Mahmood, ${ }^{2,4}$ Aman-ur-Rehman, ${ }^{2}$ \\ and S. Poedts ${ }^{1}$ \\ ${ }^{1}$ Centre for Mathematical Plasma Astrophysics, KU Leuven, Celestijnenlaan 200B, 3001 Leuven, Belgium \\ ${ }^{2}$ Pakistan Institute of Engineering and Applied Sciences, P. O. Nilore, Islamabad 45650, Pakistan \\ ${ }^{3}$ Institut für Theoretische Physik, Lehrstuhl IV: Weltraum- und Astrophysik, Ruhr-Universität Bochum, \\ D-44780 Bochum, Germany \\ ${ }^{4}$ Theoretical Physics Division, PINSTECH, P.O. Nilore, Islamabad 44000, Pakistan
}

(Received 15 August 2016; accepted 10 February 2017; published online 1 March 2017)

\begin{abstract}
The kinetic theory of electrostatic twisted waves' instability in a dusty plasma is developed in the presence of orbital angular momentum of the helical (twisted) electric field in plasmas with kappa distributed electrons, ions, and dust particles. The kappa distributed electrons are considered to have a drift velocity. The perturbed distribution function and helical electric field are decomposed by Laguerre-Gaussian mode functions defined in cylindrical geometry. The Vlasov-Poisson equation is obtained and solved analytically to investigate the growth rates of the electrostatic twisted waves in a non-thermal dusty plasma. The growth rates of the dust ion acoustic twisted mode (DIATM) and dust acoustic twisted mode (DATM) are obtained analytically and also pictorial presented numerically. The instability condition for the DIATM and DATM is also discussed with different plasma parameters. The growth rates of DIATM and DATM are larger when the drifted electrons are non-Maxwellian distributed and smaller for the Maxwellian distributed drifted electrons in the presence of the helical electric field. Published by AIP Publishing.
\end{abstract}

[http://dx.doi.org/10.1063/1.4977446]

\section{INTRODUCTION}

The helically phased light inherits orbital angular momentum (OAM). ${ }^{1}$ The work by Allen $^{2}$ on lasers with orbital angular momentum (OAM) has originated an attractive scientific and technological development in a wide range of growing fields, such as microscopy ${ }^{3}$ and imaging, ${ }^{4}$ atomic and nano-particle manipulation, ${ }^{5}$ ultra-fast optical communications, ${ }^{6}$ quantum computing, ${ }^{7}$ ionospheric radar facility to observe 3D plasma dynamics in ionosphere, ${ }^{8}$ photonic crystal fibre, ${ }^{9}$ OAM entanglement of two photons, ${ }^{10}$ twisted gravitational waves, ${ }^{11}$ ultra intense twisted laser pulses, ${ }^{12}$ and astrophysics. $^{13}$

In recent years, the study of wave propagation in plasmas carrying orbital angular momentum has appealed to the plasma community. Verbeeck and Schattschneider ${ }^{14}$ studied the production and application of electron vortex beams. The magnetic tornadoes ${ }^{15}$ (rotating magnetic field structures in the solar atmosphere) or chromospheric swirls are reported to exhibit three types of morphology, i.e., spiral, ring, and split. Leyser ${ }^{16}$ experimentally created and pumped a radio beam with helical wave front carrying OAM into the ionospheric plasma using the High frequency Active Auroral Research Program (HAARP). The optical emission spectrum of pumped plasma turbulence shows a characteristic ring shaped morphology due to the pumped twisted radio beam. The stimulated scattering instabilities are studied in a dense quantum plasma for the coherent circularly polarized

\footnotetext{
${ }^{\text {a)} E l e c t r o n i c ~ m a i l: ~ k a s h i f . a r s h a d . b u t t @ g m a i l . c o m ~}$

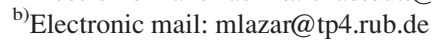

electromagnetic (CPEM) waves having orbital angular momentum. ${ }^{17}$ The Alfvenic tornadoes ${ }^{18}$ (three dimensional modified kinetic Alfven wave) have solutions such as Laguerre-Gaussian (LG) Alfvénic vortex beam. By employing Ampere's law and two fluid model, such Alfvenic tornadoes are pretended to be after the effect of plasma density whirls or magnetic flux ropes with orbital angular momentum in a magnetized plasma. Some certain scattering phenomena such as stimulated Raman and Brillouin backscattering are observed to be responsible for the interaction between electrostatic and electromagnetic waves through orbital angular momentum. ${ }^{19}$ Shukla $^{20}$ obtained the dispersive shear Alfven wave (DSAW) modes from generalized ion vorticity and electron momentum equations aligned with the magnetic field in a magnetized plasma, in Earth's auroral zone, in the solar atmosphere, ${ }^{21}$ and in a laboratory plasma, e.g., Large Plasma Device (LAPD) at the University of California, Los Angeles. $^{22}$

The twisted modes are also observed in the dusty plasma environment. Shukla ${ }^{23}$ predicted and modeled the possible propagation of dust acoustic waves as twisted vortex beams with orbital angular momentum (OAM), which can trap and transport the dust particles in plasmas. Then, braided electrostatic ion cyclotron (ESIC) modes are predicted in Saturn's F-ring, ${ }^{24}$ which has the composition of the dusty magnetoplasma and modulational instability in the form of a vortex beam (when solar wind excites the 3D kinetic Alfven wave).

Nowadays, some new features of orbital angular momentum (OAM) ${ }^{25}$ have been studied in plasmas, e.g., the generation of waves carrying OAM with a dielectric 
resonator antenna (DRA) array, ${ }^{26}$ bump on tail twisted instability in cold atomic clouds, ${ }^{27}$ and damping of the twisted waves in a Maxwellian distributed multi-component dusty plasma. $^{28}$

Charged dust particles and dusty plasmas ${ }^{29}$ are rampant in cosmic and astrophysical environments ${ }^{30}$ (interstellar-media, molecular dusty clouds, star forming dust clouds, Eagle nebula, and supernovae remnants), our solar system ${ }^{31}$ (planetary ring systems and interplanetary media due to the presence of cometary dust particles), and Earth's mesosphere. ${ }^{32}$

In laboratory experiments and most of the astrophysical environments, ${ }^{33}$ the charged particles exhibit non-Maxwellian or non-thermal distribution. ${ }^{34}$ These non-Maxwellian distributions are well reproduced by the power-laws or the so-called kappa distribution functions, which were recently applied for modeling the twisted plasma waves, such as Langmuir (electron) twisted waves and ion acoustic twisted waves. ${ }^{35}$ In space, permeating plasmas, ${ }^{36}$ streaming plasmas, ${ }^{37}$ and drifting plasmas $^{38}$ exhibit various novel instabilities in the presence of neutral and charged dust particles.

In the last decades, numerous linear and non-linear studies have been proposed to investigate ion acoustic and acoustic waves in dusty plasmas, ${ }^{39}$ for example, experimental study of dust ion acoustic waves, dispersion relation of dust acoustic waves, and non-linear dust acoustic waves. Recently, some new features were included in the study of dust acoustic and dust ion acoustic waves such as ion acoustic waves in cosmic dusty plasmas, ${ }^{40}$ spherical dust ion acoustic waves, ${ }^{41}$ and Bohm criterion in dusty plasmas, dusty clusters, and non-degenerate quantum dusty plasmas. $^{42,43}$ Akram et $a l .{ }^{26}$ have studied the generation of radio waves carrying orbital angular momentum (OAM) or vortex waves using a dielectric resonator antenna array that has relevance to the storage devices. Also notice that twisted ion acoustic and acoustic waves have been investigated for dusty plasma conditions, see Ref. 28, but restricting to standard Maxwellian distribution to describe the plasma components and without any drift of the electrons.

In this manuscript, the kinetic theory is applied to study the dust ion acoustic and dust acoustic waves in the presence of the electric field carrying orbital angular momentum in a plasma containing non-Maxwellian drifted electrons, ions, and dust particles described by the generalized Lorentzian or kappa distribution functions. The manuscript is composed of various sections. Section II contains a basic set of equations (Vlasov equation, Poisson equation, and Paraxial equation) for the twisted dust ion acoustic and dust acoustic waves. In Section III, the modified plasma dispersion function with an electric field having orbital angular momentum is derived by decomposing the potential and distribution function in Laguerre-Gaussian (LG) modes. By using the modified dispersion function, the dielectric function of twisted dust ion acoustic and dust acoustic waves in plasmas with nonthermal electrons and ions with immobile and mobile dust particles, the growth rates of the electrostatic twisted waves in nonthermal dusty plasmas are calculated analytically in Sections IV and V, respectively. In Section VI, the numerical plots for growth rates of the instabilities of electrostatic twisted waves in nonthermal dusty plasmas are illustrated and conclusion is also presented in the same section.

\section{THEORETICAL MODEL}

In this section, the basic set of dynamic equations is presented to describe the kinetic theory of electrostatic waves in a dusty plasma carrying orbital angular momentum. We can obtain the distribution function $f_{\alpha}(\mathbf{r}, \mathbf{v}, t)$ from the wellknown Vlasov equation, where $\alpha$ is the species index such that $\alpha=e, i$, and $d$ for the electrons, the ions, and dust particles. The distribution function $f_{\alpha}(\mathbf{r}, \mathbf{v}, t)$ of the electrostatic waves in a dusty plasma separates into two parts, a background part $f_{\alpha 0}$ and a perturbed part $\widehat{f}_{\alpha}$ given by the expression $f_{\alpha}=f_{\alpha_{0}}+\widehat{f_{\alpha}}$ by using the perturbation analysis. Now, we will apply linearization on the Vlasov equation with respect to perturbation, which gives us

$$
\left(\frac{\partial}{\partial t}+\mathbf{v} \cdot \frac{\partial}{\partial \mathbf{r}}\right) \widehat{f}_{\alpha}=q_{\alpha} \mathbf{E} \cdot \frac{\partial f_{\alpha 0}}{\partial \mathbf{p}_{\alpha}},
$$

where $\mathbf{p}_{\alpha}$ represents the momentum such that $\mathbf{p}_{\alpha}=m_{\alpha} \mathbf{v}$ and $\mathbf{E}$ is the electric field $\mathbf{E}=-\nabla \phi$. The electric field can be calculated from the linearized Poisson equation given below

$$
\nabla^{2} \phi=4 \pi \sum_{\alpha=e, i, d} q_{\alpha} \int \widehat{f}_{\alpha}(\mathbf{v}) d \mathbf{v} .
$$

Consider the propagation of the electrostatic waves with slowly varying amplitude along the $\mathrm{z}$-axis, i.e., $\exp (i k z)$, such that

$$
\nabla^{2} \phi=\left(\partial_{\perp}^{2}-k^{2}+2 i k \partial_{z}\right) \phi .
$$

Here, $\partial_{\perp}^{2}$ is the transverse Laplacian operator, and it can be written in the cylindrical coordinate system as $\partial_{\perp}^{2}$ $=(1 / r) \partial / \partial r(r \partial / \partial r)+\left(1 / r^{2}\right) \partial^{2} / \partial \theta^{2}$. The gradual variation of the field along the $\mathrm{z}$-axis is only possible if $\partial_{z}^{2} \phi \ll 2 k \partial_{z} \phi$. Under these conditions, the wave has a finite orbital angular momentum corresponding to the potential $\phi$, and they satisfy the paraxial equation given by

$$
\left(\partial_{\perp}^{2}+2 i k \partial_{z}\right) \phi=0 .
$$

The existence of these waves is such that they must satisfy the Poisson equation (2), which gives

$$
-k^{2} \phi=4 \pi \sum_{\alpha=e, i, d} q_{\alpha} \widehat{f}_{\alpha}(\mathbf{v}) d \mathbf{v} .
$$

Here, $q_{e}=-e, q_{i}=e$, and $q_{d}=Z_{d} e$ (where $e$ is the electronic charge and $Z_{d}$ is the dust charge number). Equations (1)-(5) are the basis of the dust ion acoustic and dust acoustic waves in plasmas with the electric field carrying orbital angular momentum.

\section{LORENTZIAN PLASMA DISPERSION FUNCTION IN A DUSTY PLASMA WITH ORBITAL ANGULAR MOMENTUM OF THE ELECTRIC FIELD}

We consider the solutions of the paraxial equation (4) for the calculation of the modified kappa distributed plasma 
dispersion function in the presence of the electric field carrying orbital angular momentum. These solutions of the paraxial equation (4) can be obtained by the superposition of Laguerre-Gaussian (LG) functions described in cylindrical coordinate system $\mathbf{r}=(r, \theta, z)$. By definition of LaguerreGaussian (LG) function $F_{p l}(r, z)$ (which is described in Ref. 44 with its properties as well), the modified potential $\phi(\mathbf{r}, t)$ can be written as follows:

$$
\phi(\mathbf{r}, t)=\sum_{p l} \widetilde{\phi}_{p l} F_{p l}(r, z) e^{i l \theta} e^{i k z-i \omega t} .
$$

Here, $\widetilde{\phi}_{p l}$ is the mode amplitude and integers $p$ and $l$ are the radial and angular mode numbers, while $\theta$ denotes the azimuthal angle. Using the spatial structure of the modified potential described in Eq. (6), the helical electric field can be written in terms of $k_{\text {eff }}$ (effective wave number) as $\mathbf{E}$ $=-i \mathbf{k}_{\text {eff }} \phi$, where $k_{\text {eff }}$ is defined by the following expression:

$$
\mathbf{k}_{e f f}=-\frac{i}{F_{p l}} \partial_{r} F_{p l} \mathbf{e}_{r}+\frac{l}{r} \mathbf{e}_{\theta}+\left(k-\frac{i}{F_{p l}} \partial_{z} F_{p l}\right) \mathbf{e}_{z} .
$$

Therefore, the helical electric field can also be resolved into its components as follows:

$$
\begin{gathered}
E_{r}=-\frac{\partial \phi}{\partial r}=-\frac{i}{F_{p l}} \partial_{r} F_{p l}, \\
E_{\theta}=-\frac{1}{r} \frac{\partial \phi}{\partial \theta}=-\frac{i l}{r} \phi
\end{gathered}
$$

and

$$
E_{z}=-\frac{\partial \phi}{\partial z}=-\left(i k+\frac{1}{F_{p l}} \partial_{z} F_{p l}\right) \phi
$$

The above set of Equations (8)-(10) confirms that the electric field exhibits helical structures instead of straight lines (as in the case of ordinary plane wave solutions) along the z-direction. But in accordance with the paraxial approximation, the axial component of the electric field is still dominant. In order to obtain the solution of coupled VlasovPoisson equations (1), (5), we will decompose the perturbed distribution function in Laguerre-Gaussian (LG) modes such that

$$
\widetilde{f}_{\alpha}(\mathbf{v})=\sum_{p l} \widetilde{f}_{p l}(\mathbf{v}) F_{p l}(r, z) e^{i l \theta} e^{i k z-i \omega t} .
$$

Further, we will substitute the decomposed distribution function (11) into the standard Vlasov equation (1). The resultant equation is then multiplied by $F_{p l}$ and integrated in $r d r$ using the orthogonality condition described in Ref. 44. The final form of the resultant equation is given as

$$
\widetilde{f}_{p l}=\sum_{\alpha=e, i, d} \frac{q_{\alpha}}{m_{\alpha}} \frac{\widetilde{\phi}_{p l}}{(x+i y)}\left(\mathbf{q}_{e f f} \cdot \partial_{\mathrm{v}} f_{\alpha 0}\right),
$$

here $x$ and $y$ can be defined as

$$
x=\left(\omega-k v_{z}\right)-l q_{\theta} v_{\theta}, \quad y=\left(q_{r} v_{r}+q_{z} v_{z}\right),
$$

such that

$$
x+i y=\left(\omega-\mathbf{q}_{e f f} \cdot \mathbf{v}\right)
$$

and

$$
\mathbf{q}_{e f f}=-i q_{r} \widehat{\mathbf{e}}_{r}+l q_{\theta} \widehat{\mathbf{e}}_{\theta}+\left(k-i q_{z}\right) \widehat{\mathbf{e}}_{z} .
$$

Here, the parameters $q_{j}$ and $q_{\theta}$ are defined as

$$
q_{j}=\int_{0}^{\infty} F_{p l} \partial_{j} F_{p l} r d r, \quad q_{\theta}=\int_{0}^{\infty} F_{p l}^{2} d r,
$$

where $j=r$ and $z$. We will now use the value of decomposed perturbed distribution function $\widetilde{f}_{p l}$ from (12) into the Poisson equation (5) to get the general form of the plasma dielectric function. We can write the plasma dielectric function $\epsilon\left(\omega, k, l q_{\theta}\right)$ for the twisted electrostatic waves in a dusty plasma as follows:

$$
\epsilon\left(\omega, k, l q_{\theta}\right)=1+\sum_{\alpha=e, i, d} \frac{\omega_{p \alpha}^{2}}{k^{2}} \int \frac{\mathbf{q}_{e f f} \cdot \partial_{\mathbf{v}} f_{0 \alpha}}{\left(\omega-\mathbf{q}_{e f f} \cdot \mathbf{v}\right)} d \mathbf{v},
$$

where $\chi\left(\omega, k, l q_{\theta}\right)$ is the susceptibility of electrostatic waves in the dusty plasma, which is defined as

$$
\chi\left(\omega, k, l q_{\theta}\right)=\sum_{\alpha=e, i, d} \frac{\omega_{p \alpha}^{2}}{k^{2}} \int \frac{\mathbf{q}_{e f f} \cdot \partial_{\mathbf{v}} f_{0 \alpha}}{\left(\omega-\mathbf{q}_{e f f} \cdot \mathbf{v}\right)} d \mathbf{v},
$$

here $\omega_{p \alpha}=\sqrt{4 \pi n_{\alpha 0}^{2} q_{\alpha}^{2} / m_{\alpha}}$ is the plasma frequency of the $\alpha$ species. Therefore, Eq. (17) can be written as

$\epsilon\left(\omega, k, l q_{\theta}\right)=1+\chi_{e}\left(\omega, l q_{\theta}, k\right)+\chi_{i}\left(\omega, l q_{\theta}, k\right)+\chi_{d}\left(\omega, l q_{\theta}, k\right)$.

The above form of the dielectric function in Equation (19) is similar to that of the usual dielectric function with planar electric field perturbations with an additional factor of azimuthal wave number $l q_{\theta}$ appearing due to the presence of orbital angular momentum of the electric field. From Eq. (17), we can write the modified condition of the Landau resonance in the presence of the helical (twisted) electric field in comparison to the planar electric field case as follows:

$$
\omega=k v_{z}+l q_{\theta} v_{\theta} \pm i\left(q_{r} v_{r}+q_{z} v_{z}\right) .
$$

The imaginary part $y=\left(q_{r} v_{r}+q_{z} v_{z}\right)$ can be neglected for understanding the physical meaning of the modified resonance condition, which is valid only if $\left(\left|q_{r}\right|,\left|q_{z}\right| \ll\left|q_{\theta}\right|\right)$ is satisfied. The modified resonance condition $\omega=k v_{z}+l q_{\theta} v_{\theta}$ resembles the Landau cyclotron resonance for the magneto plasma case. ${ }^{45}$

The susceptibility of twisted electrostatic waves in the unmagnetized dusty plasma carrying orbital angular momentum can be written in a more simplified form as

$$
\chi\left(\omega, l q_{\theta}, k\right)=\sum_{\alpha=e, i, d} \frac{\omega_{p \alpha}^{2}}{k^{2}} \int \frac{\mathbf{q}_{e f f} \cdot \partial_{\mathbf{v}} f_{0 \alpha} d \mathbf{v}}{\left(\omega-k v_{z}-l q_{\theta} v_{\theta}\right)} .
$$


Using Eq. (15), the modified plasma dispersion function $Z_{\kappa, l q_{\theta}}\left(\xi_{z \alpha}, \xi_{\theta \alpha}\right)$ for the species is defined as

$$
\begin{aligned}
Z_{\kappa, l q_{\theta}}\left(\xi_{z \alpha}, \xi_{\theta \alpha}\right)= & \sum_{\alpha=e, i, d}\left[\frac{d v_{z}}{u_{z}-v_{z}}\right. \\
& \left.\times \frac{\partial f_{0 \alpha}}{\partial v_{z}}+\frac{d v_{\theta}}{u_{\theta}-v_{\theta}} \frac{\partial f_{0 \alpha}}{\partial v_{\theta}}\right],
\end{aligned}
$$

here

$$
\xi_{z \alpha}=u_{z} / \theta_{\alpha} \quad \text { and } \quad \xi_{\theta \alpha}=u_{\theta} / \theta_{\alpha}
$$

and

$$
u_{z}=\left(\frac{\omega}{k}-\frac{l q_{\theta} v_{\theta}}{k}\right) \quad \text { and } \quad u_{\theta}=\left(\frac{\omega}{l q_{\theta}}-\frac{k v_{z}}{l q_{\theta}}\right) .
$$

Substituting Eq. (21) in Eq. (19), the plasma dielectric function can be written as

$$
\begin{aligned}
\epsilon\left(\omega, k, l q_{\theta}\right)= & 1+\frac{\omega_{p e}^{2}}{k^{2}} Z_{\kappa, l q_{\theta}}\left(\xi_{z e}, \xi_{\theta e}\right) \\
& +\frac{\omega_{p i}^{2}}{k^{2}} Z_{\kappa, l q_{\theta}}\left(\xi_{z i}, \xi_{\theta i}\right)+\frac{\omega_{p d}^{2}}{k^{2}} Z_{\kappa, l q_{\theta}}\left(\xi_{z d}, \xi_{\theta d}\right) .
\end{aligned}
$$

The three dimensional non-Maxwellian (kappa) distribution function for the electrons is given by the expression ${ }^{44}$

$$
\begin{aligned}
f_{0 e}= & \frac{n_{e 0}}{\pi^{3 / 2} \theta_{e}^{3}} \frac{\Gamma\left(\kappa_{e}+1\right)}{\kappa_{e}^{3 / 2} \Gamma\left(\kappa_{e}-1 / 2\right)} \\
& \times\left[1+\frac{1}{\kappa_{e} \theta_{e}^{2}}\left[\left(v_{z}-v_{0 e}\right)^{2}+v_{r}^{2}+v_{\theta}^{2}\right]\right]^{-\kappa_{e}-1},
\end{aligned}
$$

where $\kappa_{e}$ is the electron's spectral index and $\theta_{e}=\left(2 \kappa_{e}\right.$ $\left.-3 / \kappa_{e}\right)^{1 / 2} v_{T_{e}}$ with $\kappa>3 / 2$ the Lorentzian thermal velocity for non-thermal electrons such that $v_{T_{e}}=\left(T_{e} / m_{e}\right)^{1 / 2}$, the thermal velocity of the electrons. The electron's temperature $T_{e}$ is in energy units, appearing in the thermal velocity, and $v_{0 e}$ is the drift velocity of the electrons along the $z$ direction. The three dimensional kappa distribution function for the ions and dust particles is given as

$f_{0 i}=\frac{n_{i 0}}{\pi^{3 / 2} \theta_{i}^{3}} \frac{\Gamma\left(\kappa_{i}+1\right)}{\kappa_{i}^{3 / 2} \Gamma\left(\kappa_{i}-1 / 2\right)}\left[1+\frac{1}{\kappa_{i} \theta_{i}^{2}}\left(v_{z}^{2}+v_{r}^{2}+v_{\theta}^{2}\right)\right]^{-\kappa_{i}-1}$,

and

$f_{0 d}=\frac{n_{d 0}}{\pi^{3 / 2} \theta_{d}^{3}} \frac{\Gamma\left(\kappa_{d}+1\right)}{\kappa_{d}^{3 / 2} \Gamma\left(\kappa_{d}-1 / 2\right)}\left[1+\frac{1}{\kappa_{d} \theta_{d}^{2}}\left(v_{z}^{2}+v_{r}^{2}+v_{\theta}^{2}\right)\right]^{-\kappa_{d}-1}$,

where $\kappa_{i}$ and $\kappa_{d}$ are the spectral indices of ions and dust particles and $\theta_{i}$ and $\theta_{d}$ are the Lorentzian thermal velocities of ions and dust particles, respectively, which can be written in mathematical form as

$$
\begin{aligned}
\theta_{i} & =\left(\frac{2 \kappa_{i}-3}{\kappa_{i}}\right)^{1 / 2} v_{T_{i}} ; \quad v_{T_{i}}=\frac{T_{i}}{m_{i}} \\
\theta_{d} & =\left(\frac{2 \kappa_{d}-3}{\kappa_{d}}\right)^{1 / 2} v_{T_{d}} ; \quad v_{T_{d}}=\frac{T_{d}}{m_{d}} .
\end{aligned}
$$

We will study the dust ion acoustic twisted mode (DIATM) and dust acoustic twisted mode (DATM) in detail in Sections IV and V, respectively.

\section{DUST ION ACOUSTIC TWISTED MODE (DIATM)}

In this section, we have considered a three-component non-Maxwellian (kappa distributed) plasma consisting of inertialess electrons, inertial ions, and stationary dust particles in the background of ions and electrons. Therefore, in such plasmas, the temperature of the electrons and mass of the ions are important for the study of the well-known Dust Ion Acoustic Mode (DIAM). In our study, we have chosen a helical (twisted) electric field instead of the conventional planar electric field that will impart an azimuthal wave number to the wave's expression due to the inclusion of the its orbital angular momentum (OAM). This will generate a new mode called as the Dust Ion Acoustic Twisted Mode (DIATM). The background charge neutrality condition for the dusty plasma system is

$$
n_{e 0}+Z_{d} n_{d 0}=n_{i 0},
$$

where $n_{e 0}, n_{i 0}$, and $n_{d 0}$ are the background number densities of the electrons, ions, and dust particles, respectively, and $Z_{d}$ is the dust charge number. In order to investigate the dispersion properties of the waves and Landau wave-particle interaction: Landau damping or growth rate of the DIATM, we will start with the respective Lorentzian distributed dielectric function of DIATM, which is given as follows:

$$
\begin{aligned}
\epsilon\left(\omega, k, l q_{\theta}\right)= & 1+\frac{2 \omega_{p e}^{2}}{k^{2} \theta_{e}^{2}}\left[\frac{2 \kappa_{e}-1}{\kappa_{e}}+\xi_{z_{e}} Z\left(\xi_{z_{e}}\right)+\xi_{\theta_{e}} Z\left(\xi_{\theta_{e}}\right)\right] \\
& +\frac{2 \omega_{p_{i}}^{2}}{k^{2} \theta_{i}^{2}} \times\left[\frac{2 \kappa_{i}-1}{\kappa_{i}}+\xi_{z_{i}} Z\left(\xi_{z_{i}}\right)+\xi_{\theta_{i}} Z\left(\xi_{\theta_{i}}\right)\right],
\end{aligned}
$$

where $Z\left(\xi_{z_{e}}\right)\left[Z\left(\xi_{\theta_{e}}\right)\right]$ and $Z\left(\xi_{z_{i}}\right)\left[Z\left(\xi_{\theta_{i}}\right)\right]$ are the planar [non-planar] dispersion functions of electrons and ions and the terms $\left(\xi_{z_{e}}, \xi_{\theta_{e}}, \xi_{z_{i}}\right.$, and $\left.\xi_{\theta_{i}}\right)$ have the following expressions:

$$
\begin{aligned}
& \xi_{z_{e}}=\frac{1}{\theta_{e}}\left(\frac{\omega}{k}-v_{0 e}\right) ; \quad \xi_{\theta_{e}}=\frac{1}{\theta_{e}} \frac{\omega}{l q_{\theta}}, \\
& \xi_{z_{i}}=\frac{1}{\theta_{i}}\left(\frac{\omega}{k}\right) ; \quad \xi_{\theta_{\mathrm{i}}}=\frac{1}{\theta_{\mathrm{i}}} \frac{\omega}{\mathrm{lq}_{\theta}} .
\end{aligned}
$$

The detailed analytical calculations of $Z\left(\xi_{z_{e}}\right),\left[Z\left(\xi_{\theta_{e}}\right)\right]$ and $Z\left(\xi_{z_{i}}\right)\left[Z\left(\xi_{\theta_{i}}\right)\right]$ are given in Appendix B. We can write the above dielectric function in the form of susceptibilities of electrons and ions as $\epsilon\left(\omega, k, l q_{\theta}\right)=1+\chi_{e}+\chi_{i}$. Due to the higher temperature and smaller mass of the electrons as compared to the ions, the electrons are treated as inertialess 
species. So, we can expand the dispersion functions of the electrons in the lower and higher limits of the drifted ordinary phase velocity $\left(\omega / k-v_{0 e}\right)$ and azimuthal phase velocity $\left(\omega / l q_{\theta}\right)$ of electrons in comparison to the electron's Lorentzian thermal velocity $\theta_{e}$, i.e., $\xi_{z_{e}} \ll 1$ and $\xi_{\theta_{e}} \gg 1$. By applying the low limit for the drifted phase velocity and high limit for the azimuthal phase velocity, the expansion of the dispersion function will result in the following form of electron's susceptibility:

$$
\begin{aligned}
\chi_{e}= & \frac{2 \omega_{p e}^{2}}{k^{2} \theta_{e}^{2}}\left[\frac{2 \kappa_{e}-1}{\kappa_{e}}+i \frac{\sqrt{\pi} \kappa_{e} !}{\kappa_{e}^{3 / 2} \Gamma\left(\kappa_{e}-1 / 2\right)} \frac{1}{\theta_{e}}\left(\frac{\omega}{k}-v_{0 e}\right)\right. \\
& \left.+i \frac{\sqrt{\pi} \kappa_{e} !}{\kappa_{e}^{3 / 2} \Gamma\left(\kappa_{e}-1 / 2\right)} \frac{1}{\theta_{e}}\left(\frac{\omega}{l q_{\theta}}\right) \times\left[1+\frac{\omega^{2}}{\kappa_{e} l^{2} q_{\theta}^{2} \theta_{e}^{2}}\right]^{-\kappa_{e}-1}\right] .
\end{aligned}
$$

The ion dynamics are important here that is why its mass $m_{i}$ appears in the acoustic speed. Therefore, the dispersion relation of the ions is expanded in the higher limits of the ordinary phase velocity $(\omega / k)$ and azimuthal phase velocity $\left(\omega / l q_{\theta}\right)$ in comparison to the ion's Lorentzian velocity $\theta_{i}$, i.e., $\xi_{z_{i}} \gg 1$ and $\xi_{\theta_{i}} \gg 1$. Under these limits, the expansion of the dispersion function will result in the following form of the ions' susceptibility:

$$
\begin{aligned}
\chi_{i}= & \frac{2 \omega_{p_{i}}^{2}}{k^{2} \theta_{i}^{2}}\left[-\frac{1 k^{2} \theta_{i}^{2}}{\omega^{2}}\left(1+\frac{k^{2} \theta_{i}^{2}}{\omega^{2}} \frac{3 \kappa_{i}}{2 \kappa_{i}-3}\right)\right. \\
& +i \sqrt{\pi} \frac{\kappa_{i} !}{\kappa_{i}^{3 / 2} \Gamma\left(\kappa_{i}-1 / 2\right)} \frac{1}{\theta_{i}} \frac{\omega}{k}\left[1+\frac{\omega^{2}}{\kappa_{i} k^{2} \theta_{i}^{2}}\right]^{-\kappa_{i}-1} \\
& -\frac{1}{2} \frac{l^{2} q_{\theta}^{2} \theta_{i}^{2}}{\omega^{2}}\left(1+\frac{l^{2} q_{\theta}^{2} \theta_{i}^{2}}{\omega^{2}} \frac{3 \kappa_{i}}{2 \kappa_{i}-3}\right) \\
& \left.+i \sqrt{\pi} \frac{\kappa_{i} !}{\kappa_{i}^{3 / 2} \Gamma\left(\kappa_{i}-1 / 2\right)} \frac{1}{\theta_{i}} \frac{\omega}{l q_{\theta}}\left[1+\frac{\omega^{2}}{\kappa_{i} l^{2} q_{\theta}^{2} \theta_{i}^{2}}\right]^{-\kappa_{i}-1}\right] .
\end{aligned}
$$

It may be noted from Eqs. (33) and (34) that the dielectric function $\epsilon\left(\omega, k, l q_{\theta}\right)$ (defined in Eq. (31)) is the combination of real $\operatorname{Re}\left[\epsilon\left(\omega, k, l q_{\theta}\right)\right]$ and imaginary $\operatorname{Im}\left[\epsilon\left(\omega, k, l q_{\theta}\right)\right]$ parts. So, we can write $\epsilon\left(\omega, k, l q_{\theta}\right)=\operatorname{Re}\left[\epsilon\left(\omega, k, l q_{\theta}\right)\right]+\operatorname{Im}\left[\epsilon\left(\omega, k, l q_{\theta}\right)\right]$. In order to formulate the dispersion relation of the DIATM, we will equate the real part of the dielectric function $\operatorname{Re}\left[\epsilon\left(\omega, k, l q_{\theta}\right)\right]$ to zero, which gives

$$
\begin{aligned}
\frac{\omega_{r}^{2}}{\omega_{p_{i}}^{2}}= & \left(\frac{n_{i 0}}{n_{e 0}}\right) \frac{k^{2} \lambda_{D e}^{2}}{\Upsilon^{2}}\left[\left(\frac{\kappa_{e}-3 / 2}{\kappa_{e}-1 / 2}\right) \times \frac{1+\Upsilon^{2}}{1+k^{2} \lambda_{\kappa e}^{2}}\right. \\
& \left.+3 \frac{n_{e 0}}{n_{i 0}} \frac{T_{i}}{T_{e}}\left(\frac{1+\Upsilon^{4}}{1+\Upsilon^{2}}\right)\right],
\end{aligned}
$$

where $\Upsilon=k / l q_{\theta}$ is the ratio of the conventional $(k)$ and azimuthal wave number $\left(l q_{\theta}\right)$ and $\lambda_{\kappa e}$ the modified Debye (screening) length defined in Eq. (27) of Ref. 46, i.e.,

$$
\lambda_{\kappa e}=\left(\frac{\kappa_{e}-3 / 2}{\kappa_{e}-1 / 2}\right)^{1 / 2} \lambda_{D e} .
$$

In the limit $\Upsilon \rightarrow \infty$, Eq. (35) transforms to Eq. (28) of Ref. 46. By using the charge neutrality condition, we can write the modified equation for the dispersion relation of DIATM

$$
\begin{aligned}
\omega_{r}^{2}= & \frac{1}{\Upsilon^{2}} \frac{k^{2} C_{s}^{2}}{\left(1-\eta Z_{d}\right)} \times\left[\frac{\kappa_{e}-3 / 2}{\kappa_{e}-1 / 2} \frac{1+\Upsilon^{2}}{1+k^{2} \lambda_{\kappa e}^{2}}\right. \\
& \left.+3 \sqrt{1-\eta Z_{d}} \frac{T_{i}}{T_{e}}\left(\frac{1+\Upsilon^{4}}{1+\Upsilon^{2}}\right)\right] .
\end{aligned}
$$

Here, $\eta=n_{d 0} / n_{i 0}$ is the ratio of background number densities of dust particles to ions. By using $\eta=0$, Eq. (37) retains the expression of Eq. (34) of Ref. 35. The above dispersion relation can also be written in terms of the Dust Ion Acoustic Speed $\left(C_{D I A}\right)$, which can be defined as

$$
C_{D I A}=\omega_{p_{i}} \lambda_{D e}=\frac{C_{s}}{\sqrt{1-\eta Z_{d}}} .
$$

If the temperature of the electrons is taken much larger in comparison to the temperature of the ions, i.e., $T_{i} \ll T_{e}$, and using the above definition of the dust ion acoustic speed $\left(C_{D I A}\right)$, Equation (37) of the dispersion relation of the DIATM becomes

$$
\omega_{r}^{2}=\frac{k^{2} C_{D I A}^{2}}{\Upsilon^{2}}\left[\left(\frac{\kappa_{e}-3 / 2}{\kappa_{e}-1 / 2}\right) \frac{1+\Upsilon^{2}}{1+k^{2} \lambda_{\kappa e}^{2}}\right] .
$$

The instability condition of the DIATM in the presence of drifted electrons can be calculated from the relation of the growth rate $(\gamma){ }^{33}$ The estimation of the growth rate $(\gamma)$ can be made by putting the values of real $\operatorname{Re}\left[\epsilon\left(\omega, k, l q_{\theta}\right)\right]$ and imaginary $\operatorname{Im}\left[\epsilon\left(\omega, k, l q_{\theta}\right)\right]$ parts of the dielectric function in the relation

$$
\gamma=-\left(\frac{\partial \operatorname{Re}\left[\epsilon\left(\omega, k, l q_{\theta}\right)\right]}{\partial \omega_{r}}\right)^{-1} \operatorname{Im}\left[\epsilon\left(\omega, k, l q_{\theta}\right)\right] .
$$

By applying the above formula, the growth rate $(\gamma)$ of the DIATM can be expressed in terms of the summation of growth rates due to the Boltzmanian electrons and dynamic ions described as follows:

$$
\gamma=-\frac{\sqrt{\pi} \omega_{r}^{3} \Upsilon^{2}}{\omega_{p i}^{2}\left(1+\Upsilon^{2}\right)}\left[\gamma_{e}+\gamma_{i}\right],
$$

where $\gamma_{e}$ and $\gamma_{i}$ are the respective growth rate components of the inertialess electrons and inertial ions. The growth rate part of the electrons $\gamma_{e}$ can be further shown as the combination of the planar $\gamma_{e z}$ and azimuthal $\gamma_{e \theta}$ components as

$$
\gamma_{e}=\frac{\omega_{p e}^{2}}{k^{2} \theta_{e}^{3}}\left(\gamma_{e z}+\gamma_{e \theta}\right)
$$

such that

$$
\gamma_{e z}=\frac{\kappa_{e} !}{\kappa_{e}^{3 / 2} \Gamma\left(\kappa_{e}-1 / 2\right)}\left(\frac{\omega_{r}}{k}-v_{0 e}\right),
$$


and

$$
\gamma_{e \theta}=\frac{\kappa_{e} ! \Upsilon}{\kappa_{e}^{3 / 2} \Gamma\left(\kappa_{e}-1 / 2\right)} \frac{\omega_{r}}{k}\left(1+\Upsilon^{2} \frac{\omega_{r}^{2}}{\kappa_{e} k^{2} \theta_{e}^{2}}\right)^{-\kappa_{e}-1} .
$$

The expression of the growth rate of the ions $\gamma_{i}$ can also be written in terms of its planar $\gamma_{i z}$ and azimuthal $\gamma_{i \theta}$ components as follows:

$$
\gamma_{i}=\frac{\omega_{p_{i}}^{2}}{k^{2} \theta_{i}^{3}}\left(\gamma_{i z}+\gamma_{i \theta}\right)
$$

such that

$$
\gamma_{i z}=\frac{\kappa_{i} !}{\kappa_{i}^{3 / 2} \Gamma\left(\kappa_{i}-1 / 2\right)} \frac{\omega_{r}}{k}\left(1+\frac{\omega_{r}^{2}}{\kappa_{i} k^{2} \theta_{i}^{2}}\right)^{-\kappa_{i}-1}
$$

and

$$
\gamma_{i \theta}=\frac{\kappa_{i} ! \Upsilon}{\kappa_{i}^{3 / 2} \Gamma\left(\kappa_{i}-1 / 2\right)} \frac{\omega_{r}}{k}\left(1+\Upsilon^{2} \frac{\omega_{r}^{2}}{\kappa_{i} k^{2} \theta_{i}^{2}}\right)^{-\kappa_{i}-1} .
$$

The instability threshold condition can be obtained by writing Eq. (41) of growth rate $\gamma$ of DIATM in the nonthermal dusty plasma in a more compact form as

$$
\gamma=P\left[v_{0 e}-\frac{\omega_{r}}{k}\left(Q-\frac{t}{s} R\right)\right],
$$

where

$$
\begin{gathered}
s=\frac{\kappa_{e} !}{\kappa_{e}^{3 / 2} \Gamma\left(\kappa_{e}-1 / 2\right)} \frac{\omega_{p_{e}}^{2}}{\theta_{e}^{3}}, \\
t=\frac{\kappa_{i} !}{\kappa_{i}^{3 / 2} \Gamma\left(\kappa_{i}-1 / 2\right)} \frac{\omega_{p_{i}}^{2}}{\theta_{i}^{3}}, \\
P=\sqrt{\pi} \frac{\Upsilon^{2}}{\left(1+\Upsilon^{2}\right)} \frac{\omega_{r}^{3}}{k^{2} \omega_{p i}^{2}}, \\
Q=\left[1+\Upsilon\left(1+\Upsilon^{2} \frac{\omega_{r}^{2}}{\kappa_{e} k^{2} \theta_{e}^{2}}\right)^{-\kappa_{e}-1}\right],
\end{gathered}
$$

and

$$
\begin{aligned}
R= & {\left[\left(1+\frac{\omega_{r}^{2}}{\kappa_{i} k^{2} \theta_{i}^{2}}\right)^{-\kappa_{i}-1}\right.} \\
& \left.+\Upsilon\left(1+\Upsilon^{2} \frac{\omega_{r}^{2}}{\kappa_{i} k^{2} \theta_{i}^{2}}\right)^{-\kappa_{i}-1}\right] .
\end{aligned}
$$

The instability threshold condition of DIATM, represented by $v_{t h}$, is given as

$$
v_{t h}=v_{0 e}>\frac{\omega_{r}}{k}\left(Q-\frac{t}{s} R\right) .
$$

\section{DUST ACOUSTIC TWISTED MODE (DATM)}

This section again deals with dust acoustic wave propagation in a three-component non-Maxwellian (kappa distributed) plasma in the presence of the helical (twisted) electric field. In this study, the inertia (mass) of the dust particles is important as compared to the ions and electrons. When the dynamics of the dust particles and the temperatures of electrons and ions are crucial for the studies of the plasma mode, then such a mode is known as the Dust Acoustic Mode (DAM). This investigation includes the effect of orbital angular momentum (OAM) and azimuthal electric field, and the Dust Acoustic Mode (DAM) transforms into the Dust Acoustic Twisted Mode (DATM). To study the dispersion relation and growth rate of the DATM, we first write the following dielectric function:

$$
\begin{aligned}
\epsilon\left(\omega, k, l q_{\theta}\right)= & 1+\frac{2 \omega_{p e}^{2}}{k^{2} \theta_{e}^{2}}\left[\frac{2 \kappa_{e}-1}{\kappa_{e}}+\xi_{z_{e}} Z\left(\xi_{z_{e}}\right)+\xi_{\theta_{e}} Z\left(\xi_{\theta_{e}}\right)\right] \\
& +\frac{2 \omega_{p i}^{2}}{k^{2} \theta_{i}^{2}}\left[\frac{2 \kappa_{i}-1}{\kappa_{i}}+\xi_{z_{i}} Z\left(\xi_{z_{i}}\right)+\xi_{\theta_{i}} Z\left(\xi_{\theta_{i}}\right)\right] \\
& +\frac{2 \omega_{p d}^{2}}{k^{2} \theta_{d}^{2}}\left[\frac{2 \kappa_{d}-1}{\kappa_{d}}+\xi_{z_{d}} Z\left(\xi_{z_{d}}\right)+\xi_{\theta_{d}} Z\left(\xi_{\theta_{d}}\right)\right],
\end{aligned}
$$

here $Z\left(\xi_{z_{e}}\right)\left[Z\left(\xi_{\theta_{e}}\right)\right], Z\left(\xi_{z_{i}}\right)\left[Z\left(\xi_{\theta_{i}}\right)\right]$ and $Z\left(\xi_{z_{d}}\right)\left[Z\left(\xi_{\theta_{d}}\right)\right]$ are the planar [azimuthal] plasma dispersion functions of electrons, ions, and dust particles and the terms $\xi_{z_{e}}, \xi_{\theta_{e}}, \xi_{z_{i}}$, $\xi_{\theta_{i}}, \xi_{z_{d}}$ and $\xi_{\theta_{d}}$ have the following expressions:

$$
\begin{aligned}
& \xi_{z_{e}}=\frac{1}{\theta_{e}}\left(\frac{\omega}{k}-v_{0 e}\right) ; \quad \xi_{\theta_{e}}=\frac{1}{\theta_{e}} \frac{\omega}{l q_{\theta}}, \\
& \xi_{z_{i}}=\frac{1}{\theta_{i}}\left(\frac{\omega}{k}\right) ; \quad \xi_{\theta_{i}}=\frac{1}{\theta_{i}}\left(\frac{\omega}{l q_{\theta}}\right), \\
& \xi_{z_{d}}=\frac{1}{\theta_{d}}\left(\frac{\omega}{k}\right) ; \quad \xi_{\theta_{d}}=\frac{1}{\theta_{d}}\left(\frac{\omega}{l q_{\theta}}\right) .
\end{aligned}
$$

The detailed analytical calculations of $Z\left(\xi_{z_{e}}\right),\left[Z\left(\xi_{\theta_{e}}\right)\right]$ and $Z\left(\xi_{z_{i}}\right)\left[Z\left(\xi_{\theta_{i}}\right)\right]$ are given in Appendix B. Due to the higher temperature and smaller mass of the electrons and ions as compared to the dust particles, the dispersion functions of the electrons and ions can be expanded in the lower limit of the drifted ordinary phase velocity $\left(\omega / k-v_{0 e}\right)$ of electrons and phase velocity $(\omega / k)$ of ions and higher limit of the azimuthal phase velocity $\left(\omega / l q_{\theta}\right)$ in comparison to the electron's and ion's Lorentzian velocities $\theta_{e}$ and $\theta_{i}$, i.e., $\xi_{z_{e}}, \xi_{z_{i}} \ll 1$ and $\xi_{\theta_{e}}, \xi_{\theta_{i}} \gg 1$. The expansion of the dispersion function in the low limit for the drifted phase velocity and high limit for the azimuthal phase velocity of electrons gives the following electron's susceptibility:

$$
\begin{aligned}
\chi_{e}= & \frac{2 \omega_{p e}^{2}}{k^{2} \theta_{e}^{2}}\left[\frac{2 \kappa_{e}-1}{\kappa_{e}}+i \frac{\sqrt{\pi} \kappa_{e} !}{\kappa_{e}^{3 / 2} \Gamma\left(\kappa_{e}-1 / 2\right)} \times \frac{1}{\theta_{e}}\left(\frac{\omega}{k}-v_{0 e}\right)\right. \\
& \left.+i \sqrt{\pi} \frac{\kappa_{e} !}{\kappa_{e}^{3 / 2} \Gamma\left(\kappa_{e}-1 / 2\right)} \times \frac{1}{\theta_{e}}\left(\frac{\omega}{l q_{\theta}}\right)\left[1+\frac{\omega^{2}}{\kappa_{e} l^{2} q_{\theta}^{2} \theta_{e}^{2}}\right]^{-\kappa_{e}-1}\right],
\end{aligned}
$$


and the expansion of the dispersion function of the ions give the following ion's susceptibility:

$$
\begin{aligned}
\chi_{i}= & \frac{2 \omega_{p i}^{2}}{k^{2} \theta_{i}^{2}}\left[\frac{2 \kappa_{i}-1}{\kappa_{i}}+i \frac{\sqrt{\pi} \kappa_{i} !}{\kappa_{i}^{3 / 2} \Gamma\left(\kappa_{i}-1 / 2\right)} \times \frac{1}{\theta_{i}} \frac{\omega}{k}\right. \\
& \left.+i \sqrt{\pi} \frac{\kappa_{i} !}{\kappa_{i}^{3 / 2} \Gamma\left(\kappa_{i}-1 / 2\right)} \times \frac{1}{\theta_{i}} \frac{\omega}{l q_{\theta}}\left[1+\frac{\omega^{2}}{\kappa_{i} l^{2} q_{\theta}^{2} \theta_{i}^{2}}\right]^{-\kappa_{i}-1}\right] .
\end{aligned}
$$

It is definite about dust Lorentzian thermal velocity $\theta_{d}$ being lower in comparison to the phase velocity $(\omega / k)$ and azimuthal phase velocity $\left(\omega / l q_{\theta}\right)$ of the DATM, given that the dust is inertial while ions and electrons are inertialess. Therefore, the dispersion function of the dust particles is expanded in the limits $\xi_{z_{d}}, \xi_{\theta_{d}} \gg 1$, to find the given relation of the susceptibility of dust particles

$$
\begin{aligned}
\chi_{d}= & \frac{2 \omega_{p_{d}}^{2}}{k^{2} \theta_{d}^{2}}\left[-\frac{k^{2} \theta_{d}^{2}}{2 \omega^{2}}\left(1+\frac{k^{2} \theta_{d}^{2}}{\omega^{2}} \frac{3 \kappa_{d}}{2 \kappa_{d}-3}\right)\right. \\
& +i \sqrt{\pi} \frac{\kappa_{d} !}{\kappa_{d}^{3 / 2} \Gamma\left(\kappa_{d}-1 / 2\right)} \frac{1}{\theta_{d}} \frac{\omega}{k}\left[1+\frac{\omega^{2}}{\kappa_{d} k^{2} \theta_{d}^{2}}\right]^{-\kappa_{d}-1} \\
& -\frac{1}{2} \frac{l^{2} q_{\theta}^{2} \theta_{d}^{2}}{\omega^{2}}\left(1+\frac{l^{2} q_{\theta}^{2} \theta_{d}^{2}}{\omega^{2}} \frac{3 \kappa_{d}}{\left(2 \kappa_{d}-3\right)}\right) \\
& \left.+i \sqrt{\pi} \frac{\kappa_{d} !}{\kappa_{d}^{3 / 2} \Gamma\left(\kappa_{d}-1 / 2\right)} \frac{1}{\theta_{d}} \frac{\omega}{l q_{\theta}}\left[1+\frac{\omega^{2}}{\kappa_{d} l^{2} q_{\theta}^{2} \theta_{d}^{2}}\right]^{-\kappa_{d}-1}\right] .
\end{aligned}
$$

The susceptibilities of electrons, ions, and dust particles defined in Eqs. (58)-(60) clearly show that the dielectric function $\epsilon\left(\omega, k, l q_{\theta}\right)$ contains real and imaginary terms. So, we can write $\epsilon\left(\omega, k, l q_{\theta}\right)=\operatorname{Re}\left[\epsilon\left(\omega, k, l q_{\theta}\right)\right]+\operatorname{Im}\left[\epsilon\left(\omega, k, l q_{\theta}\right)\right]$. The dispersion relation of the Dust Acoustic Twisted Mode (DATM) can be found by solving the relation $\left(\operatorname{Re}\left[\epsilon\left(\omega, k, l q_{\theta}\right)\right]=0\right)$, which is given by

$$
\omega_{r}^{2}=\frac{k^{2} \omega_{p_{d}}^{2} \lambda_{\kappa d}^{2}}{1+k^{2} \lambda_{\kappa d}^{2}} \frac{\left(1+\Upsilon^{2}\right)}{\Upsilon^{2}}
$$

where $\lambda_{k d}$ is the Lorentzian distributed effective Debye (screening) length, which is defined as

$$
\lambda_{\kappa d}^{-2}=\left(\frac{2 \kappa_{e}-1}{2 \kappa_{e}-3}\right) \lambda_{D e}^{-2}+\left(\frac{2 \kappa_{i}-1}{2 \kappa_{i}-3}\right) \lambda_{D i}^{-2} .
$$

We can write the dispersion relation Eq. (61) in the form of the modified (Lorentzian distributed) Dust Acoustic Speed as

$$
\omega_{r}^{2}=\frac{k^{2} C_{\kappa d A}^{2}}{1+k^{2} \lambda_{\kappa d}^{2}} \frac{\left(1+\Upsilon^{2}\right)}{\Upsilon^{2}}
$$

where the modified (Lorentzian distributed) dust acoustic speed $\left(C_{\kappa d A}=\omega_{p_{d}} \lambda_{\kappa d}\right)$ has the following relation:

$$
C_{\kappa d A}^{2}=\frac{\left(\eta Z_{d}^{2}\right)\left(T_{i} / m_{d}\right)}{\left[\left(\frac{2 \kappa_{e}-1}{2 \kappa_{e}-3}\right) \frac{T_{i}}{T_{e}}\left(1-\eta Z_{d}\right)+\left(\frac{2 \kappa_{i}-1}{2 \kappa_{i}-3}\right)\right]}
$$

In order to find the growth rate of the instability due to the drift velocity of electrons, we will calculate the growth rate $\gamma$ of the DATM using real $\operatorname{Re}\left[\epsilon\left(\omega, k, l q_{\theta}\right)\right]$ and imaginary $\operatorname{Im}\left[\epsilon\left(\omega, k, l q_{\theta}\right)\right]$ values of the dielectric function $\epsilon\left(\omega, k, l q_{\theta}\right)$ defined in Eq. (58) by using the well-known relation $\gamma=-\operatorname{Im}\left[\epsilon\left(\omega, k, l q_{\theta}\right)\right]\left(\partial \operatorname{Re}\left[\epsilon\left(\omega, k, l q_{\theta}\right)\right] / \partial \omega_{r}\right)^{-1}$. Using the real and imaginary terms in the expression of $\operatorname{Im}\left[\epsilon\left(\omega, k, l q_{\theta}\right)\right]$ and $\operatorname{Re}\left[\epsilon\left(\omega, k, l q_{\theta}\right)\right]$, the growth rate $\gamma$ can be expressed as

$$
\gamma=-\frac{\sqrt{\pi} \omega_{r}^{3}}{2 \omega_{p_{d}}^{2}} \frac{\Upsilon^{2}}{\left(1+\Upsilon^{2}\right)}\left[\gamma_{e}+\gamma_{i}+\gamma_{d}\right]
$$

The factors $\gamma_{e}, \gamma_{i}$, and $\gamma_{d}$ present the contributions of the electrons, ions, and dust species to the growth rate of DATM. We can split $\gamma_{e}, \gamma_{i}$, and $\gamma_{d}$ into their planar and azimuthal components as shown in the equations given below

$$
\gamma_{e}=\frac{\omega_{p e}^{2}}{k^{2} \theta_{e}^{3}}\left[\gamma_{e z}+\gamma_{e \theta}\right] \text {. }
$$

In the above equation, $\gamma_{e}$ is the growth rate of the wave due to kappa distributed electrons in terms of its planar $\gamma_{e z}$ and azimuthal $\gamma_{e \theta}$ parts, such that

$$
\gamma_{e z}=\frac{\kappa_{e} !}{\kappa_{e}^{3 / 2} \Gamma\left(\kappa_{e}-1 / 2\right)}\left(\frac{\omega_{r}}{k}-v_{d}\right),
$$

and

$$
\gamma_{e \theta}=\frac{\kappa_{e} ! \Upsilon}{\kappa_{e}^{3 / 2} \Gamma\left(\kappa_{e}-1 / 2\right)} \frac{\omega_{r}}{k}\left(1+\Upsilon^{2} \frac{\omega^{2}}{\kappa_{e} k^{2} \theta_{e}^{2}}\right)^{-\kappa_{e}-1} .
$$

The growth of the wave due to Lorentzian distributed ions $\gamma_{i}$ in the presence of the helical electric field can also be written in terms of its longitudinal $\gamma_{i z}$ and azimuthal $\gamma_{i \theta}$ parts as

$$
\gamma_{i}=\frac{\omega_{p_{i}}^{2}}{k^{2} \theta_{i}^{3}}\left[\gamma_{i z}+\gamma_{i \theta}\right]
$$

such that

$$
\gamma_{i z}=\frac{\Gamma\left(\kappa_{i}+1\right)}{\kappa_{i}^{3 / 2} \Gamma\left(\kappa_{i}-1 / 2\right)}\left(\frac{\omega_{r}}{k}\right)
$$

and

$$
\gamma_{i \theta}=\frac{\Gamma\left(\kappa_{i}+1\right) \Upsilon}{\kappa_{i}^{3 / 2} \Gamma\left(\kappa_{i}-1 / 2\right)} \frac{\omega_{r}}{k}\left(1+\Upsilon^{2} \frac{\omega^{2}}{\kappa_{i} k^{2} \theta_{i}^{2}}\right)^{-\kappa_{i}-1} .
$$

The growth rate of the wave due to dynamics of dust particles $\gamma_{d}$ is also shown into its components $\gamma_{d z}$ and $\gamma_{d \theta}$ as follows:

$$
\gamma_{d}=\frac{\omega_{p_{d}}^{2}}{k^{2} \theta_{d}^{3}}\left[\gamma_{d z}+\gamma_{d \theta}\right]
$$




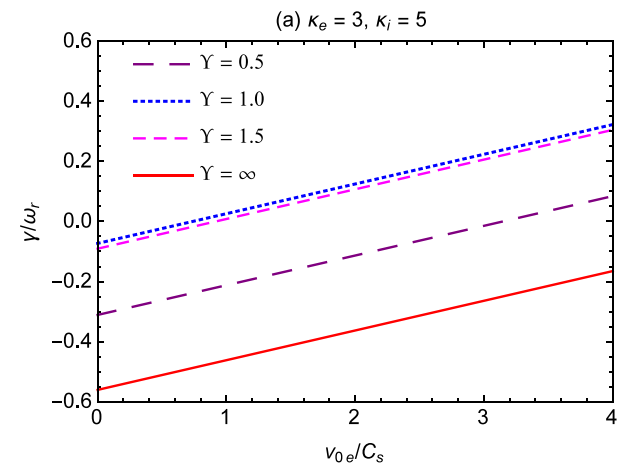

such that

$$
\gamma_{d z}=\frac{\Gamma\left(\kappa_{d}+1\right)}{\kappa_{d}^{3 / 2} \Gamma\left(\kappa_{d}-1 / 2\right)} \frac{\omega_{r}}{k}\left(1+\frac{\omega^{2}}{\kappa_{d} k^{2} \theta_{\|_{e}}^{2}}\right)^{-\kappa_{d}-1},
$$

and

$$
\gamma_{d \theta}=\frac{\Gamma\left(\kappa_{d}+1\right) \Upsilon}{\kappa_{d}^{3 / 2} \Gamma\left(\kappa_{d}-1 / 2\right)} \frac{\omega_{r}}{k}\left(1+\Upsilon^{2} \frac{\omega^{2}}{\kappa_{d} k^{2} \theta_{\|_{e}}^{2}}\right)^{-\kappa_{d}-1} .
$$

In order to calculate the instability threshold, we first write Eq. (65) in a more compact form as

$$
\gamma=-O\left[v_{0 e}-\frac{\omega_{r}}{k}\left(Q+\frac{t}{s} M+\frac{\varpi}{s} N\right)\right]
$$

where

$$
\begin{gathered}
O=\sqrt{\pi} \frac{\Upsilon^{2}}{\left(1+\Upsilon^{2}\right)} \frac{\omega_{r}^{3}}{2 k^{2} \omega_{p_{d}}^{2}}, \\
\varpi=\frac{\Gamma\left(\kappa_{d}+1\right)}{\kappa_{d}^{3 / 2} \Gamma\left(\kappa_{d}-1 / 2\right)} \frac{\omega_{p_{d}}^{2}}{\theta_{d}^{3}}, \\
M=\left[1+\Upsilon\left(1+\Upsilon^{2} \frac{\omega^{2}}{\kappa_{i} k^{2} \theta_{i}^{2}}\right)^{-\kappa_{i}-1}\right],
\end{gathered}
$$

and

$$
N=\left[\left(1+\frac{\omega^{2}}{\kappa_{d} k^{2} \theta_{\|_{e}}^{2}}\right)^{-\kappa_{d}-1}+\Upsilon\left(1+\Upsilon^{2} \frac{\omega^{2}}{\kappa_{d} k^{2} \theta_{\|_{e}}^{2}}\right)^{-\kappa_{d}-1}\right]
$$

(b) $Y=1.5$

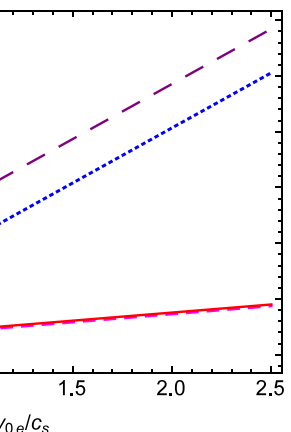

FIG. 1. The plots of normalized growth rate $\gamma / \omega_{r}$ of the Dust Ion Acoustic Twisted Mode (DIATM) are presented against the normalized electron drift velocity $v_{0 e} / C_{s}$ with $k \lambda_{\kappa e}=0.1, \eta Z_{d}$ $=0.01$, and $T_{e} / T_{i}=30$ for distinct values of (a) conventional to the azimuthal wave number $\Upsilon=k / l q \theta$ and (b) spectral indices $\kappa_{e}$ and $\kappa_{i}$.

\section{RESULTS}

In this section, we provide a detailed characterization of the instability conditions for both the Dust Ion Acoustic Twisted Mode (DIATM) and Dust Acoustic Twisted Mode (DATM). Figures 1-8 display the normalized growth rate of the unstable modes, ${ }^{47}$ enabling us to analyze the instability thresholds by tuning various parameters such as temperatures of electrons, ions, and dust, normalized electron drift velocity, and ratio of conventional to the azimuthal wave number $\Upsilon$.

\section{A. DIATM instability}

Figure 1 presents numerical plots of normalized growth rates $\gamma / \omega_{r}$ against the dimensionless electron's drift velocity $v_{0 e} / C_{s}$. In panel (a), we have considered three cases of $\Upsilon=k / l q_{\theta}$ (ratio of the parallel to the azimuthal wave number) to plot the normalized growth rate of the wave vs $v_{0 e} / C_{s}$, i.e., (i) for the azimuthal wave number $l q_{\theta}$ greater than the parallel wave number $k$ such that $\Upsilon=0.5$, (ii) when the azimuthal wave number $l q_{\theta}$ is equal to the parallel wave number $k$ such that $\Upsilon=1$, and (iii) for the azimuthal wave number $l q_{\theta}$ less than parallel wave number $k$ such that $\Upsilon=1.5$ for the non-planar (azimuthal electric field) cases, respectively, and one for the planar electric field case (iv) for the very small azimuthal wave number (so that $l q_{\theta} \cong 0$ ) in comparison to parallel wave number $k$ such that $\Upsilon=\infty$, having
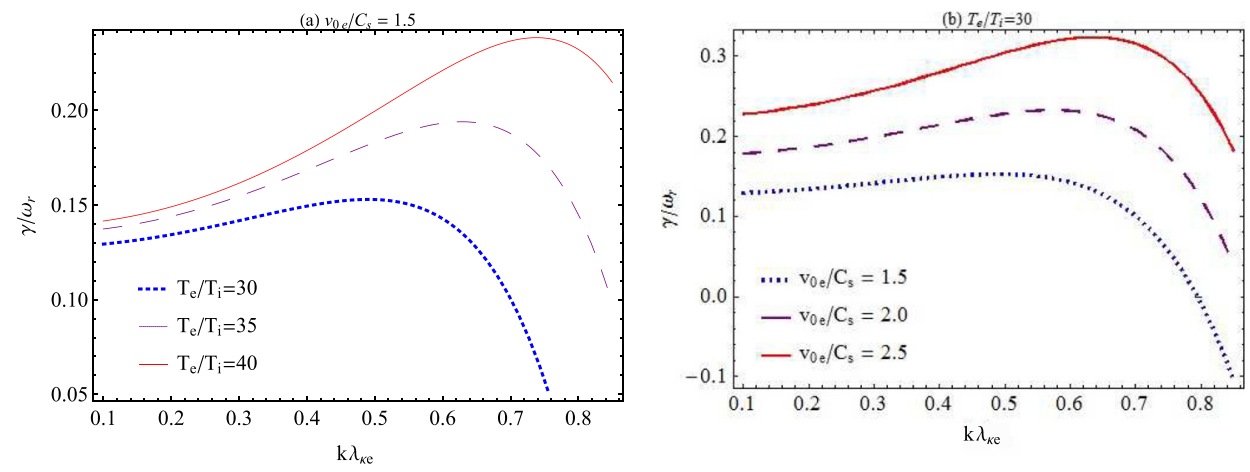

FIG. 2. The plots of normalized growth rate $\gamma / \omega_{r}$ of the Dust Ion Acoustic Twisted Mode (DIATM) are presented against the normalized wave number $k \lambda_{\text {ke }}$ with fixed values $\left(\kappa_{e}=3\right.$ and $\kappa_{i}$ $=5), \Upsilon=k / l q \theta=1, \eta Z_{d}=0.01$, and $v_{0 e} / C_{s}=1.5$ for distinct values of electron to ion temperature $T_{e} / T_{i}$ and normalized electron drift velocity $v_{0 e} / C_{s}$. 


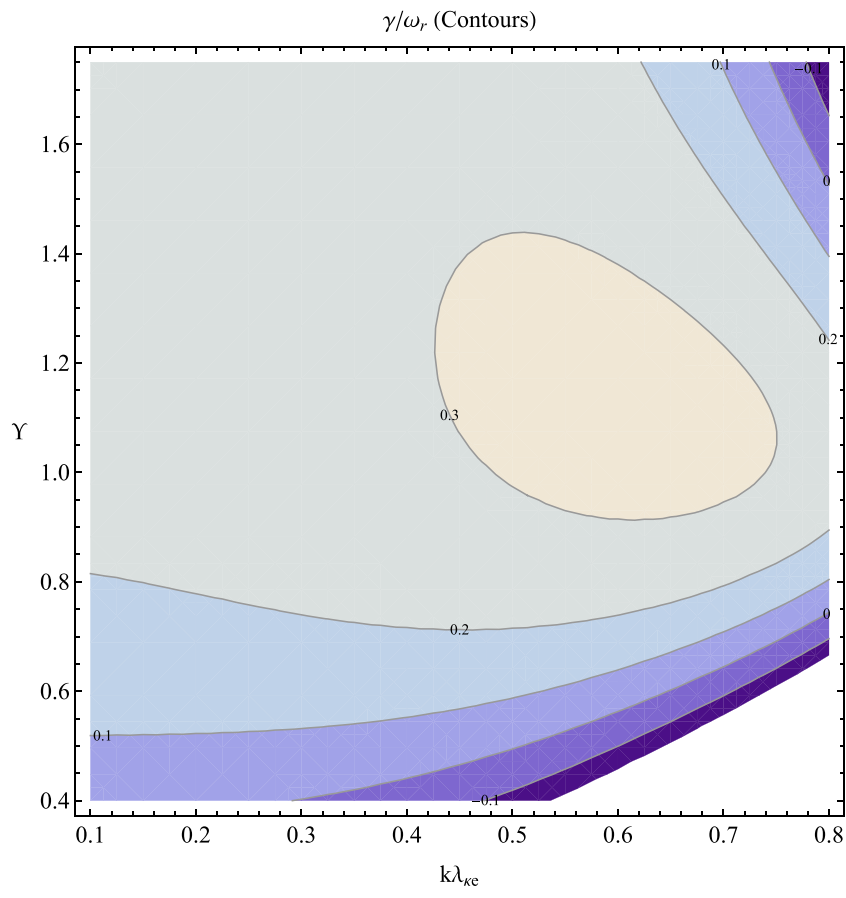

FIG. 3. The contour plots of normalized growth rate $\gamma / \omega_{r}$ of the Dust Ion Acoustic Twisted Mode (DIATM) are presented against the normalized wave number $k \lambda_{k e}$ and the ratio of conventional to the azimuthal wave number $\Upsilon=k / l q \theta$ for fixed values $\left(\kappa_{e}=3\right.$ and $\left.\kappa_{i}=5\right), v_{0 e} / C_{s}=1.5, \eta Z_{d}=0.01$, and $T_{e} / T_{i}=30$.

Lorentzian (kappa) distributed electrons and ions (i.e., $\kappa_{e}=3$ and $\kappa_{i}=5$ ). It can be observed from Figure 1(a) that the normalized growth rate of the twisted dust ion acoustic wave $\gamma /$ $\omega_{r}$ is least for $\Upsilon=0.5<1$, while smaller for $\Upsilon=1.5>1$ and larger for $\Upsilon=1$ as compared to each other for the nonplanar (azimuthal electric field) case. The physical reason of the growth rate to be large for the $\Upsilon=1.5>1$ case belongs to be that for the large azimuthal wave number the wave will take energy from more number of particles and the phenomenon of the wave particle interaction increases. Therefore, the growth of the wave for the $\Upsilon=0.5<1$ case is traced below $\Upsilon=1$ and $\Upsilon=1.5$ cases. However, if we compare the wave damping rate of the twisted wave cases $(\Upsilon=0.5,1$, and 1.5) with the planar case $(\Upsilon=\infty)$, the twisted waves are excited earlier than the planar wave.

Next, we propose to study the effects of superthermal populations by varying the values of the power-index $\kappa$. Thus, Figure 1(b) displays the growth rates for three distinct cases: (i) electrons and ions are kappa distributed (i.e., $\kappa_{e}=3$ and $\kappa_{i}=5$ ), (ii) electrons are Maxwellian distributed and

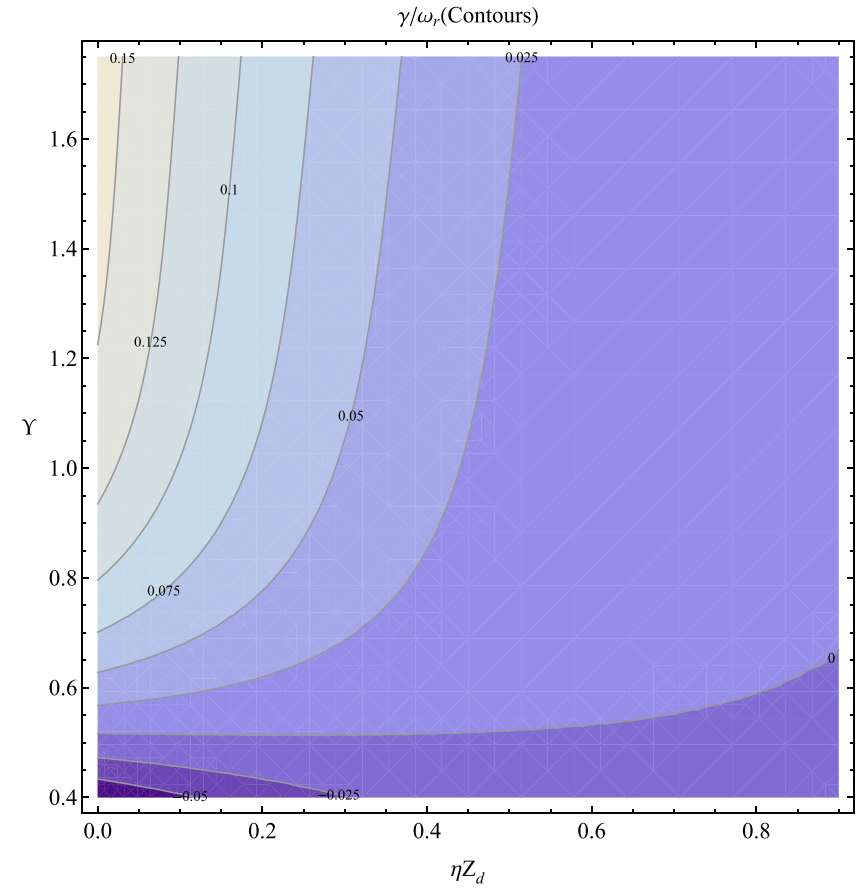

FIG. 4. The contour plots of normalized growth rate $\gamma / \omega_{r}$ of the Dust Ion Acoustic Twisted Mode (DIATM) are presented against the product of the dust charge number and number density ratio of dust to ion $\eta Z_{d}$ and the ratio of conventional to the azimuthal wave number $\Upsilon=k / l q \theta$ for fixed values $\left(\kappa_{e}=3\right.$ and $\left.\kappa_{i}=5\right), v_{0 e} / C_{s}=1.5, k \lambda_{\kappa e}=0.1$, and $T_{e} / T_{i}=30$.

ions are Kappa distributed (i.e., $\kappa_{e}=\infty$ and $\kappa_{i}=5$ ), and (iii) electrons are kappa distributed and ions are Maxwellian distributed (i.e., $\kappa_{e}=3$ and $\kappa_{i}=\infty$ ), and one case in which both electrons and ions are Maxwellian distributed (i.e., $\left.\kappa_{e}=\kappa_{i}=\infty\right)$. It can be seen from the figure that the growth rate is maximum when the inertia-less drifted electrons are kappa distributed and ions are Maxwellian distributed and the growth rate is minimum when drifted electrons are Maxwellian distributed, while the curve with kappa distributed electrons and ions lies below the maximum value and the curve with Maxwellian distributed electrons and ions lies above the minimum value. The figure clearly reveals that the growth of the instability is larger if there are more number of superthermal (kappa distributed) drifted electrons in comparison to Maxwellian distributed inertial ions. But if the superthermal nature is shared by the inertial species, then this will cause a decrease in the growth of the respective wave.

Figure 2 presents the wave number $\left(k \lambda_{\kappa e}\right)$ dispersion of the (normalized) growth rates $\gamma / \omega_{r}$. These are derived in panel (a) for three distinct values of electron to ion (a) $\kappa_{e}=\kappa_{i}=3, \kappa_{d}=10$

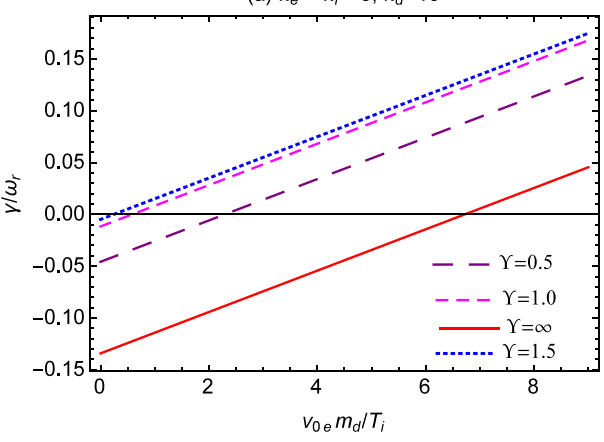

(b) $Y=1.5$

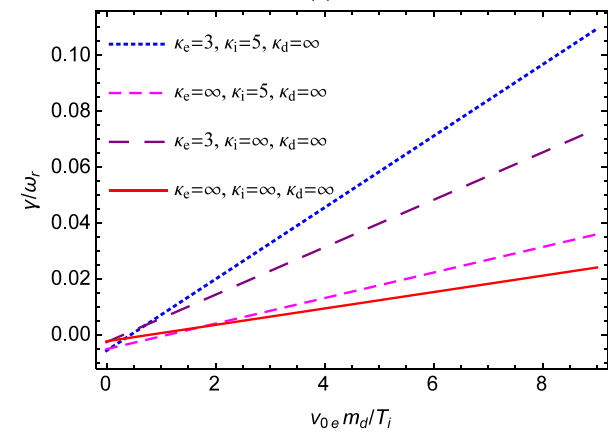

FIG. 5. The plots of normalized growth rate $\gamma / \omega_{r}$ of the Dust Acoustic Twisted Mode (DATM) are presented against the normalized electron drift velocity $v_{0 e} /\left(T_{i} / m_{d}\right)$ with $k \lambda_{\kappa d}=0.1$, $\eta Z_{d}=0.01$, and $T_{e} / T_{i}=30$ for distinct values of (a) conventional to the azimuthal wave number $\Upsilon=k / l q \theta$ and (b) spectral indices $\kappa_{e}, \kappa_{i}$, and $\kappa_{d}$. 

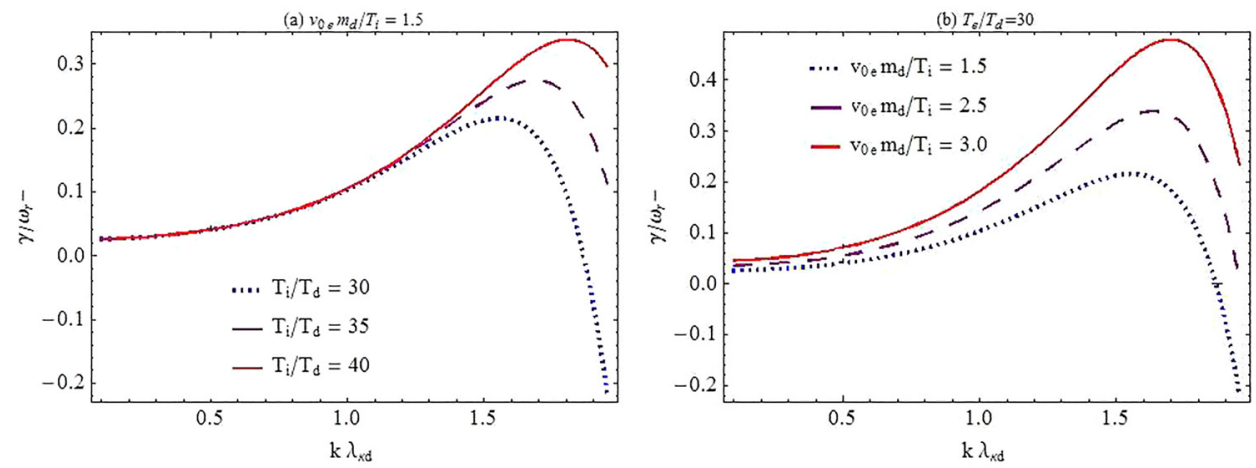

FIG. 6. The plots of normalized growth rate $\gamma / \omega_{r}$ of the Dust Acoustic Twisted Mode (DATM) are presented against the normalized wave number $k \lambda_{\kappa d}$ with fixed values $\left(\kappa_{e}=3, \kappa_{i}=3\right.$, and $\left.\kappa_{d}=10\right), \Upsilon=k / \operatorname{lq} \theta=1, \quad \eta Z_{d}=0.01$, and for distinct values of electron to ion temperature $T_{i} / T_{d}$ and normalized electron drift velocity $v_{0 e} /\left(T_{i} / m_{d}\right)$ temperature ratio $T_{e} / T_{i}$, i.e., $T_{e} / T_{i}=30,35$, and 40 . It can be seen that the normalized growth rates $\gamma / \omega_{r}$ of DIATM are minimum at $T_{e} / T_{i}=30$ and maximum at $T_{e} / T_{i}=40$. It is obvious that if the temperature of electrons is higher, then it will excite the wave more than the low value of electron temperature. Therefore, the particles that have higher temperature such that $T_{e} / T_{i}=40$ contribute more energy to the wave and resulted in the higher growth rate than for $T_{e} / T_{i}=30$. It is also observed that the growth rate of the wave first increases to the certain limit $k \lambda_{\kappa e}=0.65$, and then the decreasing trend is witnessed. The reason for this is that the wave theory remains valid for $k \lambda_{\kappa e}<1$, and this analytical study is valid for studying weak damping or growth of the wave.

In panel (b), the unstable solutions are derived for three distinct values of dimensionless electron drift velocity $v_{0 e} / C_{s}$, i.e., $v_{0 e} / C_{s}=1.5,2.0$, and 2.5 . The normalized growth rates $\gamma / \omega_{r}$ are larger at $v_{0 e} / C_{s}=2.5$ and smaller at $v_{0 e} / C_{s}=1.5$. It is evident from the figure that the higher

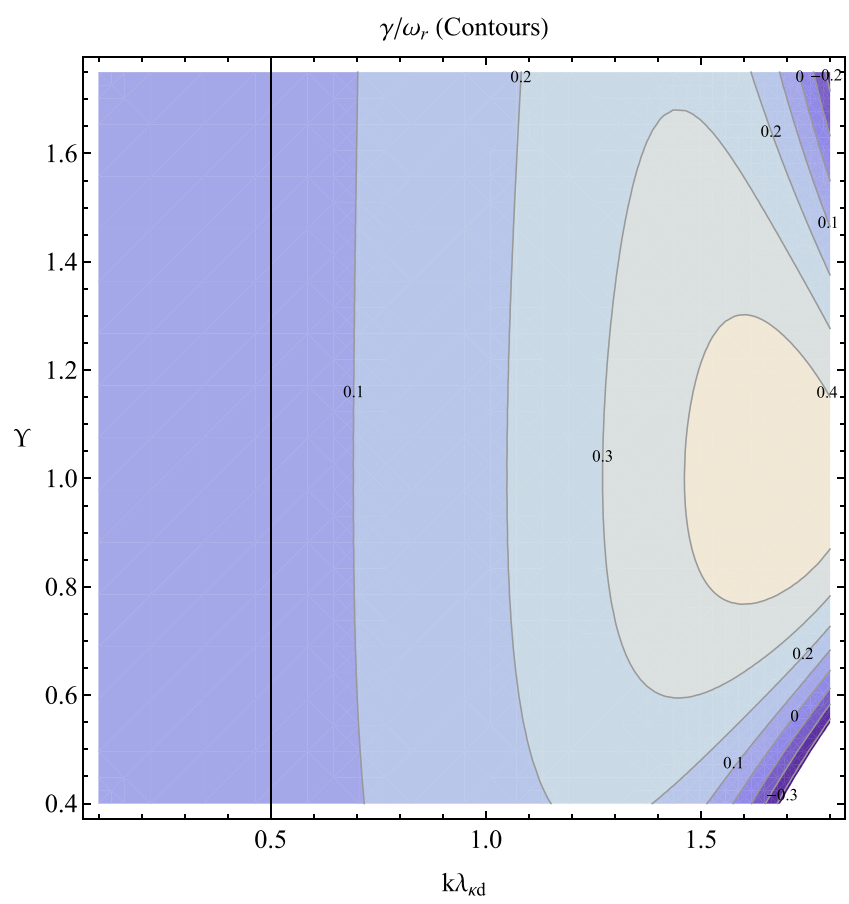

FIG. 7. The contour plots of normalized growth rate $\gamma / \omega_{r}$ of the Dust Acoustic Twisted Mode (DATM) are presented against the normalized wave number $k \lambda_{\kappa e}$ and the ratio of conventional to the azimuthal wave number $\Upsilon=k / l q \theta$ for fixed values $\left(\kappa_{e}=3, \kappa_{i}=3\right.$, and $\left.\kappa_{d}=10\right), v_{0 e} /\left(T_{i} / m_{d}\right)=1.5$, $\eta Z_{d}=0.01$, and $T_{e} / T_{i}=30$. dimensionless electron's drift velocity $v_{0 e} / C_{s}=2.5$ excites the wave at larger magnitude, and the growth rate of the wave is larger than the lower dimensionless electron's drift velocity $v_{0 e} / C_{s}=1.5$. The drift velocity acts as an energy source for the excitation and growth of the wave. If electron's drift velocity $v_{0 e} / C_{s}=2.5$ is higher, then the plasma system has a larger reservoir of the energy source to be absorbed by the wave and larger its growth rate. But the low electron's drift velocity $v_{0 e} / C_{S}=1.5$ makes a small reservoir of the energy source that can be gained by the wave through wave-particle interaction, and the resultant growth rate is small.

The contour plots of normalized growth rate $\gamma / \omega_{r}$ are shown in Figure 3 against the normalized wave numbers $\left(k \lambda_{\kappa e}\right)$ and ratio of conventional to the azimuthal wave number $\Upsilon=k / l q_{\theta}$. It is analyzed that as the value of the ratio of conventional to the azimuthal wave number $\Upsilon=k / l q_{\theta}$ increases, so do the growth rates $\gamma / \omega_{r}$. This means that the growth rate $\gamma / \omega_{r}$ is larger when the longitudinal wave

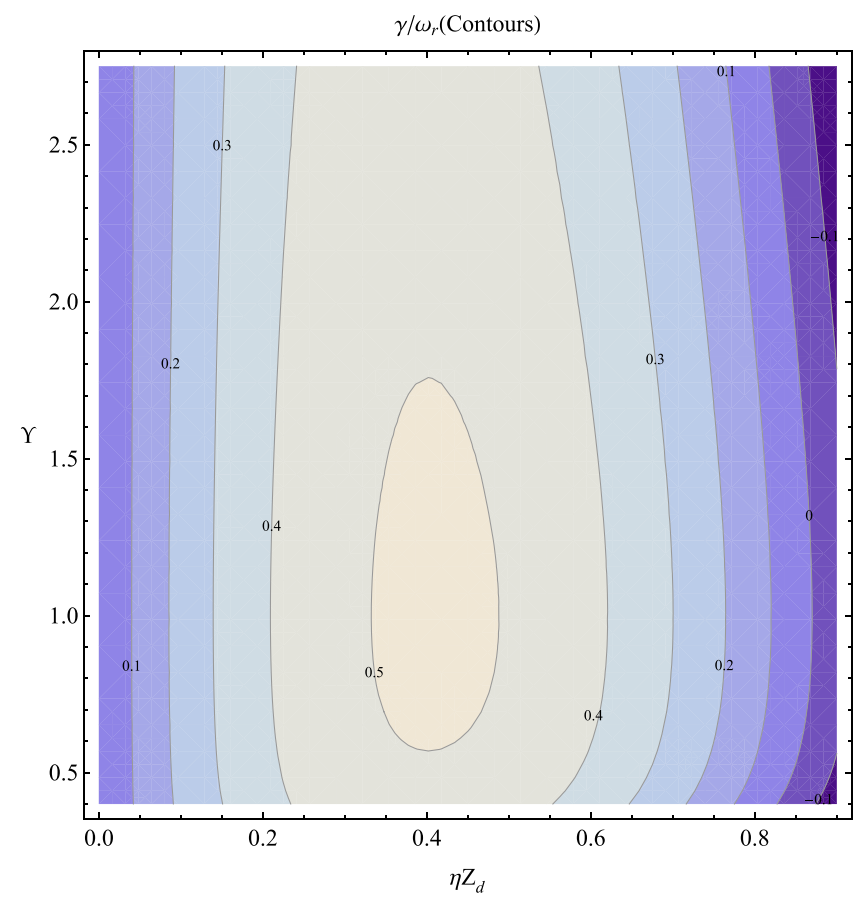

FIG. 8. The contour plots of normalized growth rate $\gamma / \omega_{r}$ of the Dust Acoustic Twisted Mode (DATM) are presented against the product of the dust charge number and number density ratio of dust to ion $\eta Z_{d}$ and the ratio of conventional to the azimuthal wave number $\Upsilon=k / l q \theta$ for fixed values $\left(\kappa_{e}=3\right.$ and $\left.\kappa_{i}=5\right), v_{0 e} /\left(T_{i} / m_{d}\right)=1.5, k \lambda_{\kappa d}=0.1$, and $T_{e} / T_{i}=30$. 
number is smaller than the azimuthal wave number (i.e., $k \ll$ $l q_{\theta}$ ) in comparison to the case when the longitudinal wave number is larger than the azimuthal wave number (i.e., $k \gg$ $\left.l q_{\theta}\right)$. The larger growth rate indicates the absorption of the greater amount of energy from the particles by the DIATM, and the smaller growth rate shows that the DIATM absorbs a lesser amount of energy from the particles during the waveparticle interaction. On the other hand, growth rate $\gamma / \omega_{r}$ increases as normalized wave number $k \lambda_{\kappa e}$ approaches the value $k \lambda_{k e} \rightarrow 4.5$, but a further increase in the wave number $k \lambda_{k e}$ leads to a decreasing trend in the growth rate; however, for large values of $k \lambda_{k e}$, the weak damping (or growth) theory of the DIATM does not remain valid.

In Figure 4, the contour plots of normalized growth rate $\gamma / \omega_{r}$ are drawn against $\eta Z_{d}$ (product of the dust charge number and number density ratio of dust to ion) and ratio of conventional to the azimuthal wave number $\left(\Upsilon=k / l q_{\theta}\right)$. It can be seen that the growth rate $\gamma / \omega_{r}$ increases with an increase in $\Upsilon$ and decreases with an increase in $\eta Z_{d}$. The longitudinal wave number $k$ increases in comparison to the azimuthal wave number $l q_{\theta}$ as $\Upsilon$ increases, which clearly reveals that if the longitudinal propagation vector $k$ is larger, then the DIATM can grow more than the situation with smaller longitudinal wave number $k$. Now, we will consider the effect of $\eta Z_{d}$ on the growth rate $\gamma / \omega_{r}$. It is understood that at larger magnitude of $\eta Z_{d}$, we have a large number of negatively charged dust particles and obviously their overall charge number is also large. So, the growth rate $\gamma / \omega_{r}$ is smaller for a large value of $\eta Z_{d}$ (in the presence of the large number of negatively charged heavy dust particles) and larger at a small value of $\eta Z_{d}$. Physically, if the number of the negatively charged dust particles increase, then the number of energetic electrons decreases that have capability to excite the wave due to their drift velocity. That is why the decreasing number density of energetic electrons causes the decrease in the excitation of the wave and hence its growth rate.

\section{B. DATM modes}

Figure 5 shows numerical plots of normalized growth rates $\gamma / \omega_{r}$ of the DATM against the normalized electron's drift velocity $v_{0 e} /\left(T_{i} / m_{d}\right)$ at various values of (a) azimuthal wave number $l q_{\theta}$ and (b) spectral parameters $\kappa_{e}, \kappa_{i}$, and $\kappa_{d}$ for the kappa distributed $\left(\kappa_{e}=3, \kappa_{i}=3\right.$, and $\left.\kappa_{d}=10\right)$ and Maxwellian distributed $\left(\kappa_{e}=\kappa_{i}=\kappa_{d} \rightarrow \infty\right)$ inertialess electrons and ions in the presence of dynamic dust. In panel (a), we have considered three cases of $\Upsilon=k / l q_{\theta}$ (ratio of the parallel to the azimuthal wave number) to draw the normalized growth rate of the wave Vs $v_{0 e} /\left(T_{i} / m_{d}\right)$, i.e., (i) for the azimuthal wave number $l q_{\theta}$ greater than the parallel wave number $k$ (i.e., $l q_{\theta}>k$ ) such that $\Upsilon=0.5$, (ii) when the azimuthal wave number $l q_{\theta}$ is equal to the parallel wave number $k$ (i.e., $l q_{\theta}=k$ ) such that $\Upsilon=1$, and (iii) for the azimuthal wave number $l q_{\theta}$ less than the parallel wave number $k$ (i.e., $l q_{\theta}<k$ ) such that $\Upsilon=1.5$ for the non-planar (azimuthal electric field) cases, respectively, and one for the planar electric field case (iv) for the very small azimuthal wave number (so that $l q_{\theta} \cong 0$ ) in comparison to the parallel wave number $k$ such that $\Upsilon=\infty$, having Lorentzian (kappa) distributed electrons, ions, and dust particles (i.e., $\kappa_{e}=3, \kappa_{i}=3$, and $\kappa_{d}=5$ ). It can be observed from the figure that the normalized growth rate of the twisted dust acoustic wave $\gamma / \omega_{r}$ is least for $\Upsilon=0.5<1$, while smaller for $\Upsilon=1.5$ and larger for $\Upsilon=1$ as compared to each other for the non-planar (azimuthal electric field) case. The physical reason of the growth rate to be large for the $\Upsilon=1.5>1$ case belongs to be that for the large azimuthal wave number the wave will take energy from more number of particles and the phenomenon of the wave particle interaction increases. Therefore, the growth of the wave for the $\Upsilon=0.5<1$ case is traced below $\Upsilon=1.5$ and $\Upsilon=1$ cases. If we compare the wave damping rate of the twisted wave cases $(\Upsilon=0.5,1$, and 1.5) with the planar case $(\Upsilon=\infty)$, the twisted waves are excited earlier than the planar waves.

In panel (b), we have considered the three cases of the Lorentzian distributed plasma such that (i) electrons and ions are kappa distributed (i.e., $\kappa_{e}=3, \kappa_{i}=5$, and $\kappa_{d}=\infty$ ), (ii) electrons are Maxwellian distributed and ions are kappa distributed (i.e., $\kappa_{e}=\infty, \kappa_{i}=5$, and $\kappa_{d}=\infty$ ), (iii) electrons are kappa distributed and ions are Maxwellian distributed (i.e., $\kappa_{e}=3, \kappa_{i}=\infty$, and $\kappa_{d}=\infty$ ), and one case in which both electrons and ions are Maxwellian distributed (i.e., $\left.\kappa_{e}=\kappa_{i}=\kappa_{d}=\infty\right)$. It can be seen from the figure that the growth rate is maximum when both electrons and ions are kappa distributed, and the growth rate is minimum when both electrons and ions are Maxwellian distributed, while the curve with kappa distributed electrons and Maxwellian distributed ions lies below the maximum value and the curve with Maxwellian distributed electrons and kappa distributed ions lies above the minimum value. The figure clearly shows that the growth rate of the DATM is larger for the kappa distributed plasma as compared to the Maxwellian distributed plasma because the kappa distributed plasma has the availability of more number of superthermal particles that can transfer their energy to the wave in comparison to the Maxwellian distributed plasma. Therefore, the wave grows more in the non-Maxwellian environment rather than in the Maxwellian scenario.

The normalized growth rates $\gamma / \omega_{r}$ of the DATM are plotted in Figure 6 against the normalized wave numbers $k \lambda_{k d}$. In panel (a), growth rates are derived for three distinct values of ion to dust temperature ratio $T_{i} / T_{d}$, i.e., $T_{i} / T_{d}=30$, 35 , and 40 . It can be seen that the normalized growth rates $\gamma / \omega_{r}$ of DATM are minimum at $T_{i} / T_{d}=30$ and maximum at $T_{i} / T_{d}=40$. It is obvious that if the temperature of ions $T_{i}$ is larger, then it will excite the wave more than the low value of ion temperature. Therefore, the ions that have higher temperature contribute more energy to the wave and result in the higher growth rate. The figure also indicates that the growth rate of the wave first increases about $k \lambda_{\kappa d}=1.5$, and then its decreasing trend is observed.

Panel (b) shows the normalized growth rates $\gamma / \omega_{r}$ against the normalized wave numbers $k \lambda_{k d}$ for three distinct values of dimensionless electron drift velocity $v_{0 e} /\left(T_{i} / m_{d}\right)$, i.e., $v_{0 e} /\left(T_{i} / m_{d}\right)=1.5,2.0$, and 2.5 . The normalized growth rates $\gamma / \omega_{r}$ are larger at $v_{0 e} /\left(T_{i} / m_{d}\right)=2.5$ and smaller at $v_{0 e} /\left(T_{i} / m_{d}\right)=1.5$. It is evident from the figure that the higher dimensionless electron's drift velocity $v_{0 e} /\left(T_{i} / m_{d}\right)$ 
$=2.5$ excites the wave at larger magnitude, and the growth rate of the wave is larger than the lower dimensionless electron's drift velocity $v_{0 e} /\left(T_{i} / m_{d}\right)=1.5$. The drift velocity acts as an energy source for the excitation and growth of the wave. If electron's drift velocity $v_{0 e} /\left(T_{i} / m_{d}\right)=2.5$ is higher, then the plasma system has a larger reservoir of the energy source to be absorbed by the wave and larger its growth rate.

The contour plots of normalized growth rate $\gamma / \omega_{r}$ are shown in Figure 7 against the normalized wave numbers $\left(k \lambda_{\kappa d}\right)$ and ratio of conventional to the azimuthal wave number $\Upsilon=k / l q_{\theta}$. It is analyzed that as the value of the ratio of conventional to the azimuthal wave number $\Upsilon=k / l q_{\theta}$ increases, so do the growth rates $\gamma / \omega_{r}$ of the DATM. This means that the growth rate $\gamma / \omega_{r}$ of the DIATM is larger when the longitudinal wave number is smaller than the azimuthal wave number (i.e., $k \ll l q_{\theta}$ ) in comparison to the case when the longitudinal wave number is larger than the azimuthal wave number (i.e., $k \gg l q_{\theta}$ ). The larger growth rate indicates the absorption of the greater amount of energy from the particles by the DATM, and the smaller growth rate shows that the DIATM absorbs a lesser amount of energy from the particles during the wave-particle interaction. On the other hand, growth rate $\gamma / \omega_{r}$ of the DATM increases as normalized wave number $k \lambda_{\kappa d}$ approaches the value $k \lambda_{\kappa d} \rightarrow 1.5$, but a further increase in wave number $k \lambda_{k d}$ causes the decreasing growth rate. The weak damping (or grow) theory of the waves in the plasma remains valid for low values of $k \lambda_{\kappa d}$, and to find growth rates of the wave for large values of normalized wave numbers, one has to do numerical analysis for studying strong damping (or growing) effects of the wave.

Figure 8 presents the contour plots of normalized growth rate $\gamma / \omega_{r}$ against $\eta Z_{d}$ (product of the dust charge number and number density ratio of dust to ion) and ratio of conventional to the azimuthal wave number $\Upsilon=k / l q_{\theta}$. It can be seen that the growth rate $\gamma / \omega_{r}$ increases with an increase in $\Upsilon$ and decreases with an increase in $\eta Z_{d}$. The longitudinal wave number $k$ increases in comparison to the azimuthal wave number $l q_{\theta}$ as $\Upsilon$ increases, which clearly reveals that if the longitudinal propagation vector $k$ is larger, then the DATM can grow more than the situation with smaller longitudinal wave number $k$. Now, we will consider the effect of $\eta Z_{d}$ on the growth rate $\gamma / \omega_{r}$ of the DATM. It is understood that at larger magnitude of $\eta Z_{d}$, we have a large number of negatively charged dust species and obviously their overall charge number is also large. So, the growth rate $\gamma / \omega_{r}$ of DATM is smaller for a large value of $\eta Z_{d}$ (in the presence of the large number of heavy dust particles) and larger at a small value of $\eta Z_{d}$. Physically meaning, the increasing number density of dust (negatively charged) particles results in the decreasing number density of energetic electrons. Now, a less number of energetic electrons are available to give energy to the wave that is why the growth rate of the wave decreases with the increasing number density of the negatively charged dust.

\section{CONCLUSION}

To conclude, we studied the kinetic description of the instability of electrostatic dust twisted modes in the in a nonthermal dusty plasmas. The electrostatic dust twisted waves in a dusty plasma were investigated using the VlasovPoisson equation, and the plasma dielectric function was derived in the presence of the orbital angular momentum (OAM) of the helical (non-planar or twisted) electric field perturbations with kappa distributed electrons, ions, and dust with distinct spectral indices. The perturbed distribution function and electric field were decomposed into LaguerreGaussian (LG) modes described in cylindrical coordinates. The growth rates of DIATM and DATM were calculated analytically using the weak damping (or grow) theory of the waves in dusty plasmas in which kappa distributed electrons were considered to be having a drift velocity. The instability conditions of the electrostatic twisted modes were also calculated analytically, and their numerical plots were also presented against appropriate physical dusty plasma parameters. The growth of the instability of DIATM and DATM is increased with the decrease in the spectral index of drifted electrons, increase in the temperature ratio of (electron to ion and ion to dust for the DIATM and DATM respectively), and dimensionless drifted velocity.

Our studies include the Lorentzian or kappa distribution function of the electrons with the shifted parallel velocity due to presence of the electron's drift in the presence of the helical electric field. The drifted electrons act as a source for the growth of the instabilities in DIATM and DATM. The threshold conditions of the instabilities for the DIATM and DATM are also calculated to estimate the minimum value of the electron's drift, which can grow the instabilities in twisted electrostatic waves in dusty plasmas. If the drift velocity of the electrons approaches to zero, then damping of DIATM and DATM is observed rather than growth. Our results can be applicable to Maxwellian distributed plasmas as well if the spectral index of the species approaches infinity. In the absence of the electron's drift velocity such that $v_{0 e}=0$ and the spectral indices $\left(\kappa_{e}=\kappa_{i}=\kappa_{d}=\infty\right)$, the real and imaginary parts of the dielectric function of DIATM and DATM resemble those given in Ref. 28. It may also be noted that we have included the non-Maxwellian or Lorentzian character of the ion and dust species along with those of electrons. The distribution of the heavier (inertial) species is usually taken as Maxwellian (thermal) if the inertialess species is non-Maxwellian distributed. In our case, we though have chosen the non-thermal (Lorentzian) distribution for the each species, but the spectral index kappa of the heavier species is larger as compared to the lighter species. Heavier particles like protons, ions, and dust particles are indeed cooler, and therefore their distributions must be closer to a Maxwellian as compared to the electrons. In natural plasmas from space, the electron distributions are well reproduced by the kappa distribution functions, which converges to a Maxwellian in the limit of very large kappa such that kappa approaches to infinity. ${ }^{34}$ It is therefore reasonable to make the assumption that the spectral index (kappa) of the heavier species is larger as compared to the lighter species. Figures 1 and 5 clearly show that the superthermal parameters or spectral indices of the species affect the growth rate of the instability, so it is wise to consider the superthermal population of each species. We have though considered the fixed 
charging of the dust particles. But sometimes, the charge of the dust fluctuates, which deviates the thermal distribution of the dust to some extent. In that case, the non-Maxwellian distribution may be the better choice than Maxwellian distribution.

\section{ACKNOWLEDGMENTS}

K.A. acknowledges support from the Katholieke Universiteit Leuven, Belgium, for hosting his research scholarship under the International Research Support Program (IRSIP) and Higher Education Commission (HEC), Pakistan, for the award of IRSIP scholarship. M.L. acknowledges support from the Katholieke Universiteit Leuven and from FWO, the Project No. G0A2316N (FWO-Vlaanderen). K.A. appreciates the support received from Shabaan Mohammed (Centre for Mathematical Plasma Astrophysics, KU Leuven) in improving the figures and the style of the manuscript.

\section{APPENDIX A: LAGUERRE-GAUSSIAN (LG) MODE FUNCTION}

- The Laguerre-Gaussian (LG) mode function $F_{p l}(r, z)$ is defined as ${ }^{35}$

$$
F_{p l}(r, z)=C_{p l} X^{|l|} L_{p}^{|l|}(X) \exp (-X / 2),
$$

where $X=r^{2} / w^{2}(z)$ such that $w(z)$ is the beam waist. Here, integers $p$ and $l$ are the radial and angular mode numbers, while $\theta$ denotes the azimuthal angle.

We can define the normalization constants $C_{p l}$ and the associated Laguerre polynomials $L_{p}^{|l|}(X)$ by the following expressions:

$$
C_{p l}=\sqrt{(l+p) ! / 4 \pi p !}
$$

and

$$
L_{p}^{|l|}(X)=\exp (X) d^{p} / d X^{p}\left[X^{l+p} \exp (-X)\right] / p ! X .
$$

The orthogonality condition for the Laguerre-Gaussian (LG) modes is given by the equation

$$
\begin{aligned}
\left\langle F_{p^{\prime} l^{\prime}} \cdot \mid F_{p l}\right\rangle= & \int_{0}^{2 \pi} d \theta \int_{0}^{\infty} r d r F_{p^{\prime} l^{\prime}} F_{p l} \\
& \times \exp \left[i\left(l-l^{\prime}\right) \theta\right]=\delta_{p p^{\prime}} \delta_{l l^{\prime}} .
\end{aligned}
$$

\section{APPENDIX B: TWISTED LORENTZIAN DISPERSION FUNCTION}

We will start the plasma dispersion function with the helical electric field carrying orbital angular momentum

$$
Z_{\kappa, l q_{\theta}}\left(\xi_{z}, \xi_{\theta}\right)=\left[\frac{d v_{z}}{u_{z}-v_{z}} \frac{\partial f_{0}}{\partial v_{z}}+\frac{d v_{\theta}}{u_{\theta}-v_{\theta}} \frac{\partial f_{0}}{\partial v_{\theta}}\right]
$$

Here, $f_{0}$ is an isotropic drifted generalized Lorentzian or kappa distribution function, which can be defined in the cylindrical coordinates as $f_{0}=\frac{1}{\pi^{3 / 2} \theta^{3} \kappa^{3 / 2}} \frac{\Gamma(\kappa+1)}{\Gamma(\kappa-1 / 2)} \times\left[1+\frac{v_{r}^{2}+v_{\theta}^{2}}{\kappa \theta^{2}}+\frac{\left(v_{z}-v_{d}\right)^{2}}{\kappa \theta^{2}}\right]^{-\kappa-1}$

By inserting the value of $\partial f_{0} / \partial v_{z}$ and $\partial f_{0} / \partial v_{\theta}$ in Eq. (B1) and taking $A_{\kappa}=\left(\pi^{3 / 2} \theta^{3} \kappa^{3 / 2}\right)^{-1} \Gamma(\kappa+1) / \Gamma(\kappa-1 / 2)$, the plasma dispersion $Z_{\kappa, l q_{\theta}}\left(\xi_{z}, \xi_{\theta}\right)$ can be written as the sum of two integrals $I_{1}$ and $I_{2}$ such that

$$
Z_{\kappa, l q_{\theta}}\left(\xi_{z}, \xi_{\theta}\right)=I_{1}+I_{2}
$$

where

$$
\begin{aligned}
I_{1}= & \frac{2(\kappa+1)}{\kappa \theta^{2}} A_{\kappa} \int_{-\infty}^{\infty} \int_{-\infty}^{\infty} \int_{-\infty}^{\infty} d v_{r} d v_{\theta} d v_{z} \frac{\left(v_{z}-v_{d}\right)}{\left(v_{z}-u_{z}\right)} \\
& \times\left[1+\frac{v_{r}^{2}+v_{\theta}^{2}}{\kappa \theta^{2}}+\frac{\left(v_{z}-v_{d}\right)^{2}}{\kappa \theta^{2}}\right]^{-\kappa-2}
\end{aligned}
$$

and

$$
\begin{aligned}
I_{2}= & \frac{2(\kappa+1)}{\kappa \theta^{2}} A_{\kappa} \int_{-\infty}^{\infty} \int_{-\infty}^{\infty} \int_{-\infty}^{\infty} d v_{r} d v_{\theta} d v_{z} \frac{v_{\theta}}{\left(v_{\theta}-u_{\theta}\right)} \\
& \times\left[1+\frac{v_{r}^{2}+v_{\theta}^{2}}{\kappa \theta^{2}}+\frac{\left(v_{z}-v_{d}\right)^{2}}{\kappa \theta^{2}}\right]^{-\kappa-2} .
\end{aligned}
$$

By performing the integration of $I_{1}$ with respect to $d v_{r}$ and using the identity

$$
\int_{0}^{\infty} e^{-(1+U) t} t^{\kappa+1} d t=\frac{\Gamma(\kappa+2)}{(1+U)^{\kappa+2}}
$$

We obtain the following expressions of $I_{1}$ :

$$
\begin{aligned}
I_{1}= & \frac{2(\kappa+1)}{\kappa \theta^{2}} A_{\kappa} \frac{\sqrt{\pi} \sqrt{\kappa} \theta \Gamma(\kappa+3 / 2)}{\Gamma(\kappa+2)} \times \int_{-\infty}^{\infty} \int_{-\infty}^{\infty} \frac{\left(v_{z}-v_{d}\right)}{\left(v_{z}-u_{z}\right)} \\
& \times\left[1+\frac{v_{\theta}^{2}}{\kappa \theta^{2}}+\frac{\left(v_{z}-v_{d}\right)^{2}}{\kappa \theta^{2}}\right]^{-\kappa-3 / 2} d v_{\theta} d v_{z} .
\end{aligned}
$$

Now, integrating $I_{1}$ with respect to $d v_{\theta}$ and using the identity

$$
\int_{0}^{\infty} e^{-(1+u) t} t^{\kappa+1 / 2} d t=\frac{\Gamma(\kappa+3 / 2)}{(1+u)^{\kappa+3 / 2}} .
$$

The expressions of $I_{1}$ becomes

$$
\begin{aligned}
I_{1}= & \frac{2(\kappa+1) \Gamma(\kappa+1)}{\Gamma(\kappa+2)} \times \pi A_{\kappa} \int_{-\infty}^{\infty} \frac{\left(v_{z}-v_{d}\right)}{\left(v_{z}-u_{z}\right)} \\
& \times\left[1+\frac{\left(v_{z}-v_{d}\right)^{2}}{\kappa \theta^{2}}\right]^{-\kappa-1} d v_{z},
\end{aligned}
$$

and finally the integration of the above integral with respect to $d v_{z}$ gives the following expression:

$$
I_{1}=\frac{2}{\theta^{2}}\left[\frac{2(\kappa-1)}{2 \kappa}+\xi_{z} Z^{*}\left(\xi_{z}\right)\right]
$$


where $Z^{*}\left(\xi_{z}\right)$ is the longitudinal component of the plasma dispersion function, which has the expression

$$
\begin{gathered}
Z^{*}\left(\xi_{z}\right)=\frac{1}{\sqrt{\pi}} \frac{\Gamma(\kappa+1)}{\kappa^{3 / 2} \Gamma(\kappa-1 / 2)} \times \int_{-\infty}^{\infty} \frac{d \varsigma_{z}}{\left(\varsigma_{z}-\xi_{z}\right)}\left[1+\frac{\varsigma_{z}^{2}}{\kappa}\right]^{-\kappa-1} \\
\varsigma_{z}=\frac{\left(v_{z}-v_{d}\right)}{\theta} ; \quad \xi_{z}=\frac{\left(u_{z}-v_{d}\right)}{\theta} .
\end{gathered}
$$

Similarly, we apply the same procedure on Eq. (B5) to get the expression

$$
I_{2}=\frac{2}{\theta^{2}}\left[\frac{2(\kappa-1)}{2 \kappa}+\xi_{\theta} Z^{*}\left(\xi_{\theta}\right)\right]
$$

and the azimuthal component of the dispersion function $Z^{*}\left(\xi_{\theta}\right)$ is represented as

$$
\begin{gathered}
Z^{*}\left(\xi_{\theta}\right)=\frac{1}{\sqrt{\pi}} \frac{\Gamma(\kappa+1)}{\kappa^{3 / 2} \Gamma(\kappa-1 / 2)} \times \int_{-\infty}^{\infty} \frac{d \varsigma_{\theta}}{\left(\varsigma_{\theta}-\xi_{\theta}\right)}\left[1+\frac{\varsigma_{\theta}^{2}}{\kappa}\right]^{-\kappa-1}, \\
\varsigma_{\theta}=\frac{\left(v_{\theta}-v_{d}\right)}{\theta} ; \quad \xi_{\theta}=\frac{\left(u_{\theta}-v_{d}\right)}{\theta} .
\end{gathered}
$$

By substituting the value of $I_{1}$ and $I_{2}$ in Eq. (B3), we obtain the given final form of the non-Maxwellian (kappa distributed) plasma dispersion function with the electric filed carrying orbital angular momentum

$$
Z_{\kappa, l q_{\theta}}\left(\xi_{z}, \xi_{\theta}\right)=\frac{2}{\theta^{2}}\left[\frac{2(\kappa-1)}{\kappa}+\xi_{z} Z^{*}\left(\xi_{z}\right)+\xi_{\theta} Z^{*}\left(\xi_{\theta}\right)\right] .
$$

${ }^{1}$ M. F. Andersen, C. Ryu, P. Clade, V. Natarajan, A. Vaziri, K. Helmerson, and W. D. Phillips, Phys. Rev. Lett. 97, 170406 (2006).

${ }^{2}$ L. Allen and M. Padgett, J. Mod. Opt. 54, 487 (2007); D. Grier, Nature 424, 810 (2003).

${ }^{3}$ A. Jesacher, S. Fürhapter, S. Bernet, and M. Ritsch-Marte, Phys. Rev. Lett. 94, 233902 (2005).

${ }^{4}$ B. Jack, J. Leach, J. Romero, and M. J. Padgett, Phys. Rev. Lett. 103, 083602 (2009).

${ }^{5}$ M. Padgett and R. Bowman, Nat. Photonics 5, 343 (2011).

${ }^{6}$ J. Wang, J. Yang, I. M. Fazal, N. Ahmed, Y. Yan, H. Huang, Y. Ren, Y. Yue, S. Dolinar, M. Tu, and A. E. Willner, Nat. Photonics 6, 488 (2012).

${ }^{7}$ G. Molina-Terriza, J. P. Torres, and L. Torner, Nat. Phys. 3, 305 (2007).

${ }^{8}$ B. Zolesi and L. R. Cander, Ionospheric Prediction and Forecasting, Springer Geophysics (Springer-Verlag, Berlin, Hiedelberg, 2014).

${ }^{9}$ H. Zhang, W. Zhang, L. Xi, X. Tang, X. Zhang, and X. Zhang, IEEE Photonics Technol. Lett. 28, 1426-1429 (2016).

${ }^{10}$ S. Oemrawsingh, X. Ma, D. Voigt, A. Aiello, E. Eliel, G.'t Hooft, and J. P. Woerdman, Phys. Rev. Lett. 95, 240501 (2005).

${ }^{11}$ I. Bialynicki-Birula and Z. Bialynicka-Birula, New J. Phys. 18, 023022 (2016).

${ }^{12}$ J. Vieira, R. M. G. M. Trines, E. P. Alves, R. A. Fonseca, J. T. Mendonca, R. Bingham, P. Norreys, and L. O. Silva, Nat. Commun. 7, 10371 (2016).

${ }^{13}$ F. Tamburini, B. Thidé, G. Molina-Terriza, and G. Anzolin, Nat. Phys. 7, 195 (2011).

${ }^{14}$ H. T. J. Verbeeck and P. Schattschneider, Nature 467, 301 (2010).

${ }^{15} \mathrm{~S}$. Wedemeyer, E. Scullion, O. Steiner, J. de la Cruz Rodriguez, and L. R. van der Voort, J. Phys.: Conf. Ser. 440, 012005 (2013).

${ }^{16}$ T. B. Leyser, L. Norin, M. McCarrick, T. R. Pedersen, and B. Gustavsson, Phys. Rev. Lett. 102, 065004 (2009).

${ }^{17}$ P. K. Shukla, B. Eliasson, and L. Stenflo, Phys. Rev. E 86, 016403 (2012).
${ }^{18}$ P. K. Shukla, J. Geophys. Res.: Space Phys. 118, 1-4, doi:10.1029/ 2012JA018247 (2013).

${ }^{19}$ J. T. Mendonca, B. Thide, and H. Then, Phys. Rev. Lett. 102, 185005 (2009).

${ }^{20}$ P. K. Shukla, Phys. Lett. A 376, 2792 (2012).

${ }^{21}$ M. V. Khotyaintsev, V. N. Melnik, B. Thide, and O. O. Konovalenko, "Combination scattering by anisotropic Langmuir turbulence with application to solar radar experiments," Sol. Phys. 234, 169 (2006); M. Khotyaintsev, "Radar probing of the Sun," Ph.D. thesis (Uppsala University, Uppsala, Sweden, 2006).

${ }^{22}$ P. Popovich, M. V. Umansky, T. A. Carter, and B. Friedman, Phys. Plasmas 17, 122312 (2010).

${ }^{23}$ P. K. Shukla, Phys. Plasmas 19, 083704 (2012).

${ }^{24}$ P. K. Shukla, Phys. Rev. E 87, 015101 (2013).

${ }^{25}$ S. M. Barnett, L. Allen, R. P. Cameron, C. R. Gilson, M. J. Padgett, F. C. Speirits, and A. M. Yao, J. Opt. 18, 064004 (2016).

${ }^{26}$ M. R. Akram, L. Gui, and Dandan Liu, in 7th Asia Pacific International Symposium on Electromagnetic Compatibility (2016).

${ }^{27}$ J. D. Rodrigues, H. Tercas, and J. T. Mendonca, Europhys. Lett. 113, 13001 (2016)

${ }^{28}$ S. Ali, S. Bukhari, and J. T. Mendonca, Phys. Plasmas 23, 033703 (2016).

${ }^{29}$ S. V. Vladirnirov, K. Ostrikov, and A. A. Samarian, Physics and Applications of Complex Plasmas (Imperial College Press, 2005), and references therein.

${ }^{30}$ K. Arshad and A. M. Mirza, Astrophys. Space Sci. 349, 753 (2014).

${ }^{31}$ I. Mann, A. Pellinen-Wannberg, E. Murad, and Z. Nemecek, Space Sci. Rev. 161, 1 (2011).

${ }^{32}$ S. Knappmiller, M. Rapp, S. Robertson, and J. Gumbel, J. Atmos. Sol.Terr. Phys. 73, 2212 (2011).

${ }^{33}$ K. Arshad and S. Mahmood, Phys. Plasmas 17, 124501 (2010); K. Arshad, S. Mahmood, and A. M. Mirza, ibid. 18, 092115 (2011); K. Arshad, Z. Ehsan, S. A. Khan, and S. Mahmood, ibid. 21, 023704 (2014).

${ }^{34}$ V. Pierrard and M. Lazar, Sol. Phys. 267, 153 (2010); M. Lazar, R. Schlickeiser, and S. Poedts, Exploring the Solar Wind (INTECH, 2012), ISBN 978-953-51-0339-4.

${ }^{35}$ K. Arshad, A. Rehman, and S. Mahmood, Phys. Plasmas 22, 112114 (2015); 23, 052107 (2016).

${ }^{36}$ K. Arshad, A. M. Mirza, and A. Rehman, Astrophys. Space Sci. 350, 585 (2014); K. Arshad, F. Siddique, A. M. Mirza, and A. Rehman, ibid.. 350, 169 (2014).

${ }^{37}$ A. Stockem Novo, P. H. Yoon, M. Lazar, R. Schlickeiser, S. Poedts, and J. Seough, Phys. Plasmas 22, 092301 (2015); M. Lazar, R. Schlickeiser, S. Poedts, and R. C. Tautz, Mon. Not. R. Astron. Soc. 390, 168 (2008); A. N. Youdin and J. Goodman, Astrophys. J. 620, 459 (2005); A. Bret, ibid. 699, 990 (2009); S. M. Khalil and W. S. Albaltan, Int. J. Astron. Astrophys. 5, 248 (2015); M. Torney, C. Mormolino, and U. De Angelis, J. Plasma Phys. 72, 189 (2006).

${ }^{38}$ R. C. Davidson, Plasma Fusion Center, Massachusetts Institute of Technology, Cambridge, Massachusetts, USA, 2014.

${ }^{39}$ A. Barkan, N. D'Angelo, and R. L. Merlino, Planet. Space Sci. 44, 239 (1996); J. B. Pieper and J. Goree, Phys. Rev. Lett. 77, 3137 (1996); A. A. Mamun, R. A. Cairns, and P. K. Shukla, Phys. Plasmas 3, 2610 (1996); F. Verheest, Planet. Space Sci. 40, 1 (1992).

${ }^{40}$ Y.-T. Gao and B. Tian, Europhys. Lett. 77, 15001 (2007); Y.-T. Gao and B. Tian, Phys. Plasmas 13, 112901 (2006).

${ }^{41}$ X. Jukui and L. He, Phys. Plasmas 10, 339 (2003); B. Tian and Y.-T. Gao, Phys. Lett. A 340, 243 (2005).

${ }^{42}$ N. Fouial, A. Tahraoui, and R. Annou, Phys. Plasmas 23, 113702 (2016); A. Bessaa and M. Djebli, ibid. 23, 113703 (2016); N. Rubab and G. Jaffer, ibid. 23, 113701 (2016).

${ }^{43}$ J. R. Heinrich, S. H. Kim, J. K. Meyer, and R. L. Merlino, Phys. Plasmas 18, 113706 (2011); W. D. Suranga Ruhunusiri and J. Goree, ibid. 21, 053702 (2014).

${ }^{44}$ M. A. Hellberg, R. L. Mace, T. K. Baluku, I. Kourakis, and N. S. Saini, Phys. Plasmas 16, 094701 (2009); G. Livadiotis, J. Geophys. Res. 120, 1607, doi:10.1002/2014JA020825 (2015); G. Livadiotis and D. J. McComas, Space Sci. Rev. 175, 183 (2013); I. Kourakis, S. Sultana, and M. A. Hellberg, Plasma Phys. Controlled Fusion 54, 124001 (2012); M. Lazar, S. Poedts, and R. Schlickeiser, Astron. Astrophys. 534, A116 (2011).

${ }^{45}$ A. F. Alexandrov, L. S. Bogdankevich, and A. A. Rukhadze, Principles of Plasma Electrodynamics (Springer-Verlag, Berlin, Heidelberg, New York, Tokio, 1984).

${ }^{46}$ T. K. Baluku and M. A. Hellberg, Phys. Plasmas 22, 083701 (2015).

${ }^{47}$ A. Iserles, A First Course in the Numerical Analysis of Differential Equations, 2nd ed. (Cambridge University Press, 2009). 